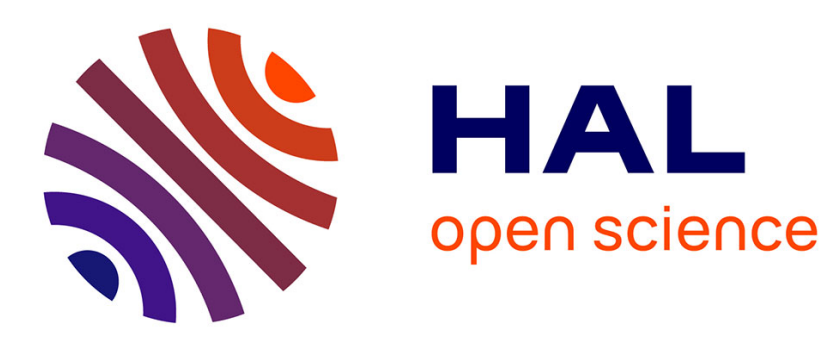

\title{
Fair management of social risk
}

Marc Fleurbaey, Stéphane Zuber

\section{To cite this version:}

Marc Fleurbaey, Stéphane Zuber. Fair management of social risk. 2016. halshs-00973480v3

\section{HAL Id: halshs-00973480 \\ https://shs.hal.science/halshs-00973480v3}

Submitted on 18 Jan 2017

HAL is a multi-disciplinary open access archive for the deposit and dissemination of scientific research documents, whether they are published or not. The documents may come from teaching and research institutions in France or abroad, or from public or private research centers.
L'archive ouverte pluridisciplinaire HAL, est destinée au dépôt et à la diffusion de documents scientifiques de niveau recherche, publiés ou non, émanant des établissements d'enseignement et de recherche français ou étrangers, des laboratoires publics ou privés. 


\section{Documents de Travail du

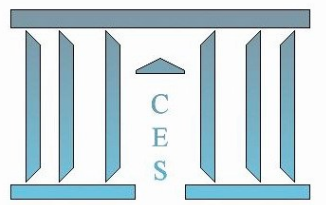

Fair management of social risk

Marc Fleurbaey, Stéphane ZuBER

2014.16RR

Version révisée

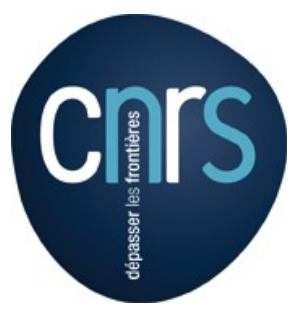

Maison des Sciences Économiques, 106-112 boulevard de L'Hôpital, 75647 Paris Cedex 13 http://centredeconomiesorbonne.univ-paris1.fr/ 


\title{
Fair management of social risk*
}

\author{
Marc Fleurbaey $^{a} \quad$ Stéphane Zuber ${ }^{b \dagger}$
}

December 2016

\begin{abstract}
We provide a general method for extending social preferences defined for riskless economic environments to the context of risk and uncertainty. We apply the method to the problems of managing unemployment allowances (in the context of macroeconomic fluctuations) and catastrophic risks (in the context of climate change). The method guarantees ex post fairness and pays attention to individuals' risk attitudes, while ensuring rationality properties for social preferences, revisiting basic ideas from Harsanyi's celebrated aggregation theorem (Harsanyi, 1955). The social preferences that we obtain do not always take the form of an expected utility criterion, but they always satisfy statewise dominance. When we require social preferences to be expected utilities, we obtain a variant of Harsanyi's result under a weak version of the Pareto principle, and a maximin criterion under a stronger Pareto requirement, whenever the ex post social ordering does not depend on people risk attitudes. We also show how non-expected utility individual preferences can be accommodated in the approach.
\end{abstract}

Keywords: Social choice, fairness, uncertainty, social risk.

JEL Classification numbers: D63.

* This work has been supported by the Agence Nationale de la Recherche (grant numbers ANR12-INEG-0006-01 and ANR-10-LABX-93); the Chair on Welfare Economics and Social Justice at the Institute for Global Studies (FMSH - Paris); the Franco-Swedish Program on Economics and Philosophy (FMSH \& Riksbankens Jubileumsfond); the Princeton Institute for International and Regional Studies.

We are very grateful to two referees and the editor for their very details comments that contributed to improve the paper. We are also grateful to Bart Capéau and audiences in Uppsala, Rotterdam, Princeton, Paris, Nanterre, Marseille, Lund, Leuven, Cergy, for comments.

${ }^{\dagger}$ Corresponding author.

${ }^{a}$ Woodrow Wilson School and University Center for Human Values, Princeton University, Princeton NJ 08544, USA. E-mail: mfleurba@princeton.edu.

b Paris School of Economics - CNRS, Centre d'Économie de la Sorbonne, 106-112 boulevard de l’Hôpital, 75013 Paris, France. E-mail: Stephane.Zuber@univ-paris1.fr. 


\section{Introduction}

Public policies are fraught with uncertainty about their consequences. To deal with the evaluation of such uncertain consequences, the literature either adopts the expected utilitarian criterion or incorporates risk and uncertainty as a simple extension of the commodity space - commodities being relabeled as contingent on the realization of a state of nature, as proposed in the framework of general equilibrium theory by Debreu (1959, chap. 7). But either approach is questionable.

The expected utilitarian criterion was famously defended by Harsanyi (1955), who proved the following theorem: if (i) all individuals and the social observer are expected utility maximizers on the set of simple acts over a set $X$; (ii) whenever all individuals prefer act $p$ to act $q$, the social observer also prefers $p$ to $q$ (the Pareto principle); then the social observer's utility function must be an affine combination of individuals' von Neumann-Morgenstern (VNM) utility functions. This result is known as Harsanyi's aggregation theorem. ${ }^{1}$ Although Harsanyi viewed this theorem as a positive result justifying utilitarianism, it is more accurate to understand it as pointing to the tension between rationality (expected utility), ex ante Pareto, and equity.

Regarding equity in resource allocation, Harsanyi's theorem is clearly problematic. Consider a one-commodity economy with two individuals 1 and 2 who face a risk on their incomes described by random variables $\tilde{x}_{1}$ and $\tilde{x}_{2}$. Assume furthermore that the individuals are expected utility maximizers so that their ordinal preferences are represented by $E u_{1}\left(\tilde{x}_{1}\right)$ and $E u_{2}\left(\tilde{x}_{2}\right)$. Harsanyi's theorem tells us that society should aim at maximizing $E\left(\alpha u_{1}\left(\tilde{x}_{1}\right)+u_{2}\left(\tilde{x}_{2}\right)\right)$, for some relative weight $\alpha$. Thus, on riskless prospects, the social welfare criterion should be $\alpha u_{1}\left(x_{1}\right)+u_{2}\left(x_{2}\right)$. If the individuals have different risk preferences, this social criterion does not satisfy the Pigou-Dalton transfer principle in general. ${ }^{2}$ If $u_{1}$ and $u_{2}$ are the same and $\alpha=1$, then the Pigou-Dalton transfer principle is satisfied but inequality aversion is constrained by risk aversion, which does not seem particularly appealing either.

Starting from a criterion of equity in resource allocation, one could think that it

\footnotetext{
${ }^{1}$ Several versions of the theorem have been established for different expected utility models in frameworks involving risk or uncertainty (Blackorby et al., 1999; Fishburn, 1984; Mongin, 1995, 1998).

${ }^{2}$ At any value $x$ such that $\alpha u_{1}^{\prime}(x) \neq u_{2}^{\prime}(x)$, a small inequality in favor of the individual with greater marginal utility is better than equality.
} 
is natural to adopt the other approach invoked in the first paragraph, dealing with contingent commodities. The problem is that such an approach is likely to entail a violation of rationality at the social level. Indeed, an act that increases inequality ex post, thereby making sure that the final consequence is worse in every state of the world, may appear attractive ex ante if everyone has a reasonable chance of being on the winning side. A typical fair criterion extended to acts in this way will therefore often violate the basic rationality condition of statewise dominance. This violation appears to be a direct consequence of the combination of the ex ante Pareto principle with fairness considerations, as illustrated in the extreme case of an egalitarian society by Gajdos and Tallon (2002). The tension between rationality, ex ante Pareto and fairness is therefore serious, and one must seek compromises that trade off these important values.

In this paper, we examine how to evaluate risky prospects while preserving basic rationality conditions (either statewise dominance or expected utility) as well as basic fairness principles. Our approach is compatible with a wide range of social criteria for the evaluation of non-risky allocations, involving various types of interpersonal comparisons and degrees of inequality aversion. In particular, we are not committed to the assumption that individuals' expected utilities provide the correct measure for assessing and comparing individual welfare. This assumption was made in the initial paper by Harsanyi (1955), and in most of the subsequent literature (Broome, 1991; Diamond, 1967; Epstein and Segal, 1992; Fleurbaey, 2010; Gajdos and Maurin, 2004; Grant et al., 2010). More recently, Adler (2012) revived the idea of using VNM utilities for the measurement of well-being in the context of evaluations with social welfare functions. But this remains a controversial assumption (as argued for instance by Chambers and Echenique, 2012) and many alternative welfare measures have been proposed in the literature, including happiness measures, capabilities and money-metric utilities (Decancq et al., 2015). The approach we propose in this paper is compatible with most of these different views.

We first show that statewise dominance and ex ante Pareto restricted to perfectly egalitarian prospects already characterize a particular way of extending a given social ordering from non-risky allocations to risky prospects. It consists in first constructing a hypothetical prospect where in each state of the world the allocation is replaced by an equally good egalitarian allocation. Then, a social welfare ordering is applied to the distribution well-being levels at the certainty-equivalent of the hypothetical egalitarian 
prospect (Proposition 1). If we strengthen the Pareto principle and apply it to the union of riskless prospects and perfectly egalitarian prospects, we show that the social ordering used to assess the certainty-equivalent of the hypothetical prospects is precisely the social ordering for non-risky allocations (Proposition 2).

These extended social orderings obtained in Propositions 1 and 2 always satisfy statewise dominance but may violate stronger rationality conditions. Therefore we examine the implications of imposing the expected utility condition on these orderings. We first show that, under the weaker ex ante Pareto principle applied to egalitarian prospects, the social VNM utility function (applied to the hypothetical equivalent egalitarian prospects) is an affine combination of individuals' VNM utility functions, much like in Harsanyi's initial result (Theorem 1). If we furthermore require the social ordering of risky prospects to satisfy a stronger Pareto requirement, and if one does not want the social ordering on riskless prospects to depend on individual risk attitudes, then it turns out that under mild assumptions this social ordering must have the maximin form (Theorem 2).

With the new class of criteria characterized in Theorem 1 (which thus encompasses the criteria obtained in Theorem 2), we revisit classical issues of risk management. We first show that the optimal management of unemployment allowances proposed by Gollier (1991) is by and large vindicated but needs adjustment in order to accommodate a special concern for income inequalities that the policy-maker may have. This may attenuate or even reverse Gollier's result that unemployment allowances should be countercyclical with wages, for some distributions of wages and some values. The opposite conclusion may be obtained because pro-cyclical allowances induce less inequalities in good states of the world. With a strong degree of priority to the worst-off, the unemployment allowances become rigid in order to provide full insurance to the unemployed.

We then revisit Weitzman's dismal theorem (Weitzman, 2009). While he studied transfers of resources to the future, we focus on the prevention of catastrophes (i.e., policies affecting probabilities rather than the consumption stream), for which a variant of the dismal theorem exists for the utilitarian criterion. We show that our criterion makes it possible to have an even greater social willingness to pay for the prevention of catastrophes if the catastrophic state is worse than the situation of the current generation, but also a lower willingness to pay in the opposite case. 
We finally examine the extension of our analysis to the case in which probabilities are not defined (a case usually labelled uncertainty rather than risk). The bulk of our analysis remains valid in this case, but new questions arise about the aggregation of beliefs. The distributive preferences embodied in the social ordering of riskless prospects have an impact on the management of ambiguity at the social level which may appear problematic. A tentative conclusion may be that the aggregation of beliefs should be separated from the aggregation of preferences and should be performed at an earlier stage.

The paper is organized as follows. In Section 2 we introduce the framework, which is Savage's state-contingent model of choice under uncertainty (Savage, 1972). Section 3 introduces a basic result showing the general implications of combining special versions of the Pareto principle with the statewise dominance principle for the social evaluation of uncertain allocations. Section 4 contains our main results, in the case in which the expected utility condition holds at the social level. In Section 5, we apply the new criterion to the optimal management of unemployment allowances and of catastrophic risks. Last, in Section 6 we explore how to extend our analysis to the context of uncertainty.

\section{The model}

We consider an economy composed of a set $N=\{1, \cdots, n\}$ of individuals. An allocation $x$ is an element of a set $X$. For instance, $X$ can be a set of allocations of $\ell$ divisible goods, which is a subset of $\mathbb{R}_{+}^{n \ell} \cdot 3$ But many other economic environments are possible, including indivisible goods alongside divisible goods.

We consider economies where the final allocation may be uncertain. Let $\Omega$ be the set of states of the world and $\mathcal{A}$ a $\sigma$-algebra over $\Omega$. A simple act $f$ is a finite-valued $\mathcal{A}$-measurable function $f: \Omega \rightarrow X$, and we denote $\mathcal{F}$ the set of simple acts. Given any $x \in X$, we abuse notation and let $x \in \mathcal{F}$ also denote the constant act such that $x(\omega)=x$ for all $\omega \in \Omega$, thus identifying $X$ with the subset of the constant acts in $\mathcal{F}$.

Until Section 6, we assume that a probability measure $p$ on the measurable space $(\Omega, \mathcal{A})$ appropriately represents the evaluator's beliefs concerning the likelihood of the

\footnotetext{
${ }^{3}$ Notation: $\mathbb{R}$ denotes the set of real numbers and $\mathbb{R}_{+}$(resp. $\mathbb{R}_{++}$) the set of non-negative (resp. positive) real numbers. $\mathbb{N}$ denotes the set of positive integers. For any $n \in \mathbb{N}$, and any set $C, C^{n}$ is the $n$-fold Cartesian product of $C$.
} 
different events in $\mathcal{A}$. In this case, we also assume that individual $i$ 's interests are appropriately represented by an expected utility. For a function $u_{i}: X \rightarrow \mathbb{R}, i \in N$ and an act $f \in \mathcal{F}$, we define the expected utility of $f$ as

$$
E\left(u_{i} \circ f\right)=\int_{\Omega} u_{i}(f(\omega)) d p(\omega) .
$$

Acts are ranked by individual $i$ through an ordering $R_{i}$ over the set $\mathcal{F}$ (an ordering is a total, antisymmetric and transitive relation). We make the following assumption on $R_{i}$.

Assumption 1 For each $i \in N$, there exists a utility function $u_{i}: X \rightarrow \mathbb{R}$ such that for all $f \in \mathcal{F}, E\left(u_{i} \circ f\right)$ is well defined, and for all $f, g \in \mathcal{F}, f R_{i} g$ if and only if $E\left(u_{i} \circ f\right) \geq E\left(u_{i} \circ g\right)$.

The problem studied in this paper is the selection of a reasonable social ordering $R_{\mathcal{F}}$ on $\mathcal{F}$. More specifically, we want to define such an ordering so as to extend a given social ordering $R_{X}$ defined over riskless allocations $X$. As explained in the introduction, the motivation for setting the question in this way is that we want our analysis to be compatible with a broad range of approaches to social evaluation and interpersonal comparisons in riskless contexts.

The social relations $R_{\mathcal{F}}$ and $R_{X}$ are orderings over $\mathcal{F}$ and $X$, respectively. We denote by $P_{\mathcal{F}}$ (resp., $I_{\mathcal{F}}$ ) the asymmetric (resp., symmetric) part of $R_{\mathcal{F}}$, and $P_{X}$ (resp., $I_{X}$ ) the asymmetric (resp., symmetric) part of $R_{X}$. Until Section 4.2, this is a singleprofile analysis: individual characteristics are given, as well as $R_{X}$.

Throughout the paper, the ordering $R_{X}$ will be assumed to have a specific structure described in Assumption 2. To state the assumption, we need to introduce a few terms. An ordering $\succsim$ over $\mathbb{R}^{n}$ is weakly monotonic, if, for all $z, z^{\prime} \in \mathbb{R}^{n}$, (a) $z \geq z^{\prime}$ implies $z \succsim z^{\prime}$; (b) $z \gg z^{\prime}$ implies $z \succ z^{\prime}$. A function $F: X \rightarrow \mathbb{R}$ is said to represent an ordering $R$ on $X$ if, for all $x, y \in X, x R y$ if and only if $F(x) \geq F(y)$.

Assumption 2 There exist functions $v_{i}: X \rightarrow \mathbb{R}$ that represent $R_{i}$ on $X$ for all $i \in N$, and a weakly monotonic ordering $R_{W}$ on $\mathbb{R}^{n}$, such that $\forall x, y \in X$,

$$
x R_{X} y \Leftrightarrow\left(v_{i}(x)\right)_{i \in N} R_{W}\left(v_{i}(y)\right)_{i \in N} .
$$

\footnotetext{
${ }^{4}$ For two vectors $x=\left(x_{1}, \cdots, x_{n}\right), y=\left(y_{1}, \cdots, y_{n}\right) \in \mathbb{R}^{n}, x \geq y$ if $x_{i} \geq y_{i}$ for all $i \in\{1, \cdots, n\}$ and $x \gg y$ if $x_{i}>y_{i}$ for all $i \in\{1, \cdots, n\}$.
} 
Assumption 2 implies that the social ordering for riskless allocations $R_{X}$ combines the construction of individual metrics $v_{i}$ that appropriately represent individual interests, and a social welfare ordering $R_{W}$ that ranks distributions of these individual measures. The $v_{i}$ numbers are the currency of social justice while $R_{W}$ embodies the conception of social justice by aggregating individual indices into an overall 'social welfare'.

We may label social orderings satisfying Assumption 2 formally welfarist in order to emphasize that the $v_{i}$ functions need not be interpreted as subjective measures of well-being, as in the welfarist tradition, and can be other measures of individual achievement. The key feature of these measures, however, is that they represent the individual orderings $R_{i}$. Note that, even though in this paper we interpret $R_{i}$ as individual preferences, they could also be interpreted as independent social judgements made on individual situations, as in Rawls' theory of primary goods (Rawls, 1971) or in Sen's theory of functionings (Sen, 1985). To simplify the exposition, we however refer to the $v_{i}$ numbers as the 'well-being levels'.

The fact that $R_{W}$ is weakly monotonic and that the $v_{i}$ functions represent individual preferences imply that the social ordering $R_{X}$ satisfies the weak Pareto principle (defined ex post on riskless allocations) that whenever $x P_{i} y$ for all $i \in N$ it must be the case that $x P_{X} y$. We do not assume the full "Strong Pareto" principle because we want our approach to be general enough to cover social preferences such as the maximin criterion.

Welfare economics has provided various methods for the construction of social orderings $R_{X}$ of riskless allocations. Most of them take the form exhibited in Equation (1), where the $v_{i}$ functions are normalized to have the same range. For instance, the Pazner-Schmeidler social ordering (Pazner, 1979) uses

$$
v_{i}(x)=\min \left\{\lambda \in \mathbb{R}_{+} \mid(\lambda z) R_{i} x_{i}\right\},
$$

where $R_{i}$ represents $i$ 's preferences over bundles of divisible goods, $x_{i} \in \mathbb{R}_{+}^{\ell}$ is the bundle allocated to individual $i$ in allocation $x$ (people have self-centered preferences), and $z$ is a reference bundle. The Pazner-Schmeidler social ordering then involves a maximin $R_{W}$ ordering. The Egalitarian Walrasian ordering (Fleurbaey and Maniquet, 2011) is a more complex example, in which individual indices depend on the whole allocation: $v_{i}(x)=\min \left\{p y_{i} \mid y_{i} R_{i} x_{i}\right\}$, for $p$ a reference price vector over divisible goods that is chosen to maximize $\min _{i} v_{i}(x) / p \mathcal{E}$, where $\mathcal{E}$ is the total endowment in the economy. In 
this example, $v_{i}(x)$ depends on the whole allocation via the choice of $p$, which typically varies with the allocation. Another example is provided by the sum of normalized VNM utilities, axiomatized by Dhillon and Mertens (1999), for which $v_{i}(x)=\frac{u_{i}(x)-\underline{u}_{i}}{\bar{u}_{i}-\underline{u}_{i}}$, where $\bar{u}_{i}:=\sup _{z \in X} u_{i}(z)$ and $\underline{u}_{i}:=\inf _{z \in X} u_{i}(z)$. A last example of normalized utilities are the health utility indices discussed in Drummond et al. (2005). They could be combined with different social welfare orderings $R_{W}$ to obtain specific criteria. In all these cases, the normalization of the range of the $v_{i}$ functions embodies an idea of impartiality and hence fair treatment of all individuals (for arguments in favor of this interpretation, see Karni, 1998; Segal, 2000).

Note that each $v_{i}(x)$ depends on the whole allocation $x$, meaning that our formalism can also cover situations with other-regarding preferences (as in Dufwenberg et al., 2011; Decerf and Van der Linden, 2016; Treibich, 2015).

Assumptions 1 and 2 imply that both $u_{i}$ and $v_{i}$ represent $R_{i}$ on $X$. Hence, they are ordinally equivalent, which means that for all $x, y \in X$,

$$
u_{i}(x) \geq u_{i}(y) \Longleftrightarrow v_{i}(x) \geq v_{i}(y)
$$

Thus there exist increasing functions $\phi_{i}$ such that $u_{i}=\phi_{i} \circ v_{i}$. We will say that the function $\phi_{i}$ represents the "risk attitude" of individual $i$ because it embodies the aversion to risk on well-being as measured by the $v_{i}$ function.

Let $X^{e}$ denote the set of (welfare) egalitarian allocations i.e., $x \in X$ such that

$$
\forall i, j \in N, v_{i}(x)=v_{j}(x)
$$

Let $\mathcal{F}^{e}$ denote the set of egalitarian acts, i.e. $\mathcal{F}^{e}=\left\{f \in \mathcal{F} \mid f(\omega) \in X^{e}\right.$ for all $\left.\omega \in \Omega\right\}$.

We now define an equally-distributed quasi-equivalent (EDQE) of an allocation $x \in X$. For any $x \in X$, let $D(x)=\left\{y \in X \mid v_{i}(y)>v_{i}(x) \forall i \in N\right\}$ be the set of allocations Pareto dominating $x$.

Definition 1 For any $x \in X, x^{e} \in X^{e}$ is an equally-distributed quasi-equivalent (EDQE) of $x$ if for all $y \in D\left(x^{e}\right)$ and for all $z \in X$ such that $x^{e} \in D(z)$,

$$
y P_{X} x \text { and } x P_{X} z
$$

Obviously, for an egalitarian allocation $x \in X^{e}$, one can take $x^{e}=x$. Note also that the EDQE allocation is generally not unique: any egalitarian allocation which is deemed 
as good as an EDQE allocation by all individuals is also an EDQE allocation. The definition of the EDQE straightforwardly extends from allocations to acts by letting, for every $f \in \mathcal{F}, f^{e}$ be defined by $f^{e}(\omega)=(f(\omega))^{e}$ for all $\omega \in \Omega$.

We make the following assumption to guarantee the existence of EDQE allocations.

Assumption 3 There exists a non-degenerate interval $V$ in $\mathbb{R}$ such that

$$
\left\{\left(w_{1}, \cdots, w_{n}\right) \in \mathbb{R}^{n} \mid \exists x \in X, v_{i}(x)=w_{i} \forall i \in N\right\}=V^{n}
$$

Assumption 3 imposes a product structure for the set of possible well-being levels. This is stronger than the condition that well-being indices are normalized to have the same range. Intuitively, it means that in the economic structure depicted by $X$, we need at least one divisible personal good that can be freely distributed to compensate other dimensions of well-being.

Lemma 1 Under Assumptions 2 and 3:

(i) For all $x \in X$, there exists $x^{e} \in X^{e}$ such that $x^{e}$ is an EDQE of $x$. Furthermore if $\tilde{x}^{e}$ is another EDQE of $x$, then $x^{e} I_{X} \tilde{x}^{e}$.

(ii) For all $x, y \in X$, if $x^{e} P_{X} y^{e}$, then $x P_{X} y$.

(iii) If $R_{W}$ is continuous, then for all $x \in X, x^{e} I_{X} x$.

Proof. See Appendix A.

When $x^{e} I_{X} x$, we call $x^{e}$ an equally-distributed equivalent (EDE) of $x$.

Let us now introduce principles that we will use to derive $R_{\mathcal{F}}$ from the social ordering of riskless allocations $R_{X}$. The first obvious principle requires the social orderings $R_{\mathcal{F}}$ and $R_{X}$ to agree on riskless allocations. It embodies our basic objective, which is to extend social orderings on riskless allocations in a consistent way.

Agreement For all $x, y \in X, x R_{\mathcal{F}} y$ if and only if $x R_{X} y$.

The second principle is a basic principle of social rationality, involving statewise dominance: if an act is better in every state of the world, it should be deemed better. ${ }^{5}$

\footnotetext{
${ }^{5}$ There is an extension of this rationality principle called eventwise dominance. It will be introduced
} 
Dominance For all $f, g \in \mathcal{F}$, if for all $\omega \in \Omega, f(\omega) R_{\mathcal{F}} g(\omega)$ then $f R_{\mathcal{F}} g$; if for all $\omega \in \Omega$, $f(\omega) P_{\mathcal{F}} g(\omega)$ then $f P_{\mathcal{F}} g$.

The next principle is a restricted version of the Pareto principle, introduced by Fleurbaey (2010) and adapted to the present framework. We do not require $R_{\mathcal{F}}$ to be fully Paretian, but only to respect unanimous preferences in the context of equality.

Pareto for equal risk For all $f, g \in \mathcal{F}^{e}$, if $f R_{i} g$ for all $i \in N$, then $f R_{\mathcal{F}} g$; if $f P_{i} g$ for all $i \in N$, then $f P_{\mathcal{F}} g$.

A key motivation for using a restricted version of the Pareto principle is that, as recalled in the introduction, the standard Pareto principle is generally incompatible with inequality aversion in the presence of Dominance. It remains, however, to explain why the restriction is to egalitarian acts. Egalitarian acts have the particular feature that risk takes a pure macroeconomic form and does not generate ex post inequalities. Therefore this restriction of Pareto is compatible with any degree of inequality aversion in the presence of Dominance.

Another motivation is that the full Pareto principle forces the evaluator to ignore the correlation between individual payoffs, making it impossible to use information about whether the social situation itself is risky or not. By restricting the Pareto principle to cases of equality (involving perfect correlation), it remains possible to use information about payoff correlation. Egalitarian acts are special because the fate of society is then perfectly aligned with individuals' fate.

Note that under Agreement, Dominance imposes $f R_{\mathcal{F}} g$ for all pairs of acts such that $f(\omega) I_{X} f\left(\omega^{\prime}\right) R_{X} g(\omega) I_{X} g\left(\omega^{\prime}\right)$ for all $\omega, \omega^{\prime} \in \Omega$. These are acts in which there is no "social" risk, although there may be individual risk. Typical examples of such acts involve negative correlation between individuals, with fixed numbers of "winners" and "losers" (as in a casino lottery). For these acts, Dominance leaves no room for Pareto and directly derives $f R_{\mathcal{F}} g$ from $R_{X}$. Egalitarian acts, with perfect correlation,

later. Note that, with our notational convention that every element $x \in X$ is identified with the constant $x$-valued act, and under agreement, we could have formulated this principle in the following way: For all $f, g \in \mathcal{F}$, if for all $\omega \in \Omega, f(\omega) R_{X} g(\omega)$ then $f R_{\mathcal{F}} g$ (and similarly for the strict preference). However, we prefer to analytically distinguish the consistency of the two social orderings (Agreement) and the internal consistency of $R_{\mathcal{F}}$ (Dominance). 
are at the opposite end of the spectrum and are those in which Dominance is the least constraining.

A last motivation for Pareto for equal risk is that we want to separately aggregate values (or tastes) and risk (or uncertainty) attitudes. The social ordering $R_{X}$, which is given, already aggregates values, and it remains to define how risky situations are to be assessed. To aggregate risk attitudes, the literature on the social aggregation of preferences under uncertainty has often resorted to applications of versions of the Pareto principle applied only to situations where people have the same preferences ex post, see for instance Cres et al. (2011), Nascimento (2012), Qu (2015) or Danan et al. (2016). We follow the same approach in this paper.

A strengthening of the above Pareto principle is Pareto for equal or no risk.

Pareto for equal or no risk For all $f, g \in \mathcal{F}^{e} \cup X$, if $f R_{i} g$ for all $i \in N$, then $p R_{\mathcal{F}} q$; if $f P_{i} g$ for all $i \in N$, then $p P_{\mathcal{F}} q$.

As explained above, one motivation to restrict the Pareto principle to egalitarian acts is that the full Pareto principle erases information about individual payoff correlation, whereas egalitarian acts display perfect coincidence between individual and social risk. Extending the Pareto principle to the comparison of riskless acts with egalitarian acts does not force ignoring payoff correlation, since this remains in the subset of acts with perfect correlation (riskless acts display perfect correlation in a degenerate way).

Moreover, in the comparison between a riskless act and an egalitarian act, if everyone prefers the risky egalitarian act, this means that even the most risk-averse among the best-off in the riskless allocation prefers the risk of equalization. Conversely, if everyone prefers to avoid the risk, this includes the least risk-averse among the worst-off in the riskless allocation, i.e., those who would potentially stand to benefit the most from the risky equalization. Therefore such unanimity still appears quite compelling, even if it may happen that, ex post, some win and some lose as a result of a unanimous choice.

Pareto for equal or no risk is already implied by Pareto for equal risk under our assumptions, when individuals are all risk neutral with respect to well-being, that is when $u_{i}=v_{i}$ for all $i \in N{ }^{6}$ We may call this the Bernoulli case, as it assumes that

\footnotetext{
${ }^{6}$ Note that the line of reasoning is still true when when all individuals have the same risk aversion with respect to the relevant wellbeing metric, that is there exists an increasing function $\phi$ such that
} 
individuals' VNM utilities provide the correct measure for assessing and comparing individual welfare. Let $f \in \mathcal{F}^{e}, x \in X$ be such that $f R_{i} x$ for all $i \in N$. This means that $E\left(u_{i} \circ f\right) \geq \max _{j} u_{j}(x)$ for all $i \in N$. By Assumption 3, there is $y \in X^{e}$ such that $u_{i}(y)=\max _{j} u_{j}(x)$ for all $i \in N$. By weak monotonicity of $R_{W}$ (Assumption 2), $y R_{X} x$, thus by Agreement, $y R_{\mathcal{F}} x$. By Pareto for equal risk, $f R_{\mathcal{F}} y$. Therefore, by transitivity, $f R_{\mathcal{F}} x$. A similar reasoning applies when $x R_{i} f$ for all $i$. The case of $f, g \in \mathcal{F}^{e}$ and the case $x, y \in X$ are implied respectively by Pareto for equal risk and Assumption 2.

Pareto for equal or no risk may thus seem to be a natural generalization of Pareto for equal risk and the Pareto property for riskless allocations implied by Agreement and Assumption 2 (more precisely, monotonicity of $R_{W}$ ). It would also be implied by a Pareto for no-reranking risk principle, defined as a version of the Pareto principle applied when individuals have the same relative positions in all states of the world (Fleurbaey, 2010). ${ }^{7}$ But although it may seem a weak strengthening of Pareto for equal risk and Pareto for riskless allocations, we show in Section 4.2 that Pareto for equal or no risk imposes strong constraints on inequality aversion when the social ordering of acts is an expected utility. This suggests that it may be difficult to find versions of the Pareto principle stronger than those studied here, but compatible with a large class of social rankings $R_{X}$ of riskless allocations.

\section{Social orderings satisfying Dominance}

\subsection{The basic results}

Our first result describes the structure of social orderings $R_{\mathcal{F}}$ satisfying Pareto for equal risk and Dominance. We show that they consist in an aggregation of the well-being levels individuals achieve at the certainty equivalent of the EDQE allocations.

To present this result, we need additional notation. For all $f \in \mathcal{F}$, let $c e(f) \in X$ denote a certainty-equivalent allocation, i.e., an allocation such that for all $i \in N$, $u_{i}=\phi \circ v_{i}$ for all $i \in N$.

${ }^{7}$ More precisely, an act $f \in \mathcal{F}$ involves no reranking if $\left(v_{i}(f(\omega))-v_{j}(f(\omega))\right)\left(v_{i}\left(f\left(\omega^{\prime}\right)\right)-\right.$ $\left.v_{j}\left(f\left(\omega^{\prime}\right)\right)\right) \geq 0$, for all $i, j \in N$ and $\omega, \omega^{\prime} \in \Omega$. By definition, egalitarian acts and riskless acts involve no reranking. For a similar idea, in the context of inequality measurement under uncertainty, see the paper by Chew and Sagi (2012), which proposes a property of mean-comotonic independence. 
$u_{i}(c e(f))=E\left(u_{i} \circ f\right)$. In light of Assumption 3, it is natural to assume that it is always possible to find such an allocation, but we need to make the assumption explicit since no topological structure was assumed for $X{ }^{8}$

Assumption 4 For all $f \in \mathcal{F}$, there is an allocation ce $(f) \in X$ such that for all $i \in N$, $u_{i}(c e(f))=E\left(u_{i} \circ f\right)$.

For an egalitarian allocation $f \in \mathcal{F}^{e}$ and $\varepsilon \in \mathbb{R}_{++}$, we denote $f-\varepsilon$ an act $f^{\prime} \in \mathcal{F}^{e}$ such that $v_{i}\left(f^{\prime}(\omega)\right)=v_{i}(f(\omega))-\varepsilon$ for all $i \in N$ and $\omega \in \Omega$ (provided such an act exists). Similarly, we denote $f+\varepsilon$ an act $f^{\prime} \in \mathcal{F}^{e}$ such that $v_{i}\left(f^{\prime}(\omega)\right)=v_{i}(f(\omega))+\varepsilon$ for all $i \in N$ and $\omega \in \Omega$ (provided such an act exists). We also let $Z=\left\{z \in \mathbb{R}^{n} \mid\right.$ $\left.\exists f \in \mathcal{F}^{e}, z=\left(v_{i}(c e(f))\right)_{i \in N}\right\}$ be the set of individual welfare distributions that can be reached at certainty-equivalents of egalitarian acts.

Proposition 1 Under Assumptions 1, 2, 3 and 4, if the social ordering $R_{\mathcal{F}}$ satisfies Agreement, Dominance and Pareto for equal risk, then there exists a weakly monotonic ordering $\succsim^{*}$ on $Z$ such that for all $f, g \in \mathcal{F}, f P_{\mathcal{F}} g$ whenever there exists $\varepsilon \in \mathbb{R}_{++}$such that

$$
\left(v_{i}\left(c e\left(f^{e}-\varepsilon\right)\right)\right)_{i \in N} \succsim^{*}\left(v_{i}\left(c e\left(g^{e}+\varepsilon\right)\right)\right)_{i \in N} .
$$

Proof. See Appendix A.

Loosely speaking, Proposition 1 describes a three-step construction of the social ordering of uncertain allocations. First, in each state of the world, we compute an EDQE allocation. Then, we compute the certainty equivalent for the individuals at the uncertain EDQE act. Last, we assess the certainty equivalent by combining well-being indices $v_{i}$ with an ordering $\succsim^{*}$ of the distribution of individuals' well-being levels. This presentation is not completely correct, because we do not compare directly the certainty equivalent of the EDQE acts, but the well-being levels from these certainty equivalent plus or minus some small amount of welfare. In particular $\left(v_{i}\left(c e\left(f^{e}\right)\right)\right)_{i \in N} \succsim^{*}\left(v_{i}\left(c e\left(g^{e}\right)\right)\right)_{i \in N}$ would not be sufficient to guarantee that $f R_{\mathcal{F}} g$, because the ordering $\succsim^{*}$ need not be continuous.

\footnotetext{
${ }^{8}$ An alternative would be to make additional structural assumptions, and for instance to assume that $X$ is connected and the $u_{i}$ functions are continuous. We prefer to avoid such assumptions to be as general as possible.
} 
Recall, however, that if $R_{W}$ is continuous, then the EDQE $x^{e}$ is a true EDE, i.e., $x^{e} I_{X} x$ (Lemma 1 (iii)). In that case Proposition 1 can be formulated in a much simpler way.

Corollary 1 Under Assumptions 1, 2, 3 and 4, if $R_{W}$ is continuous and the social ordering $R_{\mathcal{F}}$ satisfies Agreement, Dominance and Pareto for equal risk, then there exists a weakly monotonic ordering ${ }^{*}$ on $Z$ such that for all $f, g \in \mathcal{F}, f R_{\mathcal{F}} g$ if and only if

$$
\left(v_{i}\left(c e\left(f^{e}\right)\right)\right)_{i \in N} \succsim^{*}\left(v_{i}\left(c e\left(g^{e}\right)\right)\right)_{i \in N} .
$$

Proof. See Appendix A.

The social orderings described in Proposition 1 and Corollary 1 bear some resemblance with ex post social criteria obtained by Gajdos and Maurin (2004). ${ }^{9}$ They obtain a two-step procedure that first applies a social welfare function to social outcomes in each state of the world, and then computes a certainty equivalent of these uncertain social welfare levels. In the present paper, we need a third step, namely the aggregation of wellbeing levels at the certainty equivalent allocation because individuals may have different risk preferences.

Proposition 1 does not put any constraint on the ordering $\succsim^{*}$ that aggregates wellbeing levels at the certainty equivalent allocation, except weak monotonicity. Note that the ordering of the certainty equivalent of EDQE acts resembles the construction of a formally welfarist social ordering as defined in Assumption 2. But although the $v_{i}$ metric must be used, the ordering ${ }^{*}$ need not be related to the ordering $R_{W}$ used to assess allocations when there is no risk. This is in contrast with Proposition 2, which is obtained when we impose Pareto for equal or no risk. Therefore, not much can be said in general on the exact form of $R_{\mathcal{F}}$ on the sole basis of Dominance and Pareto for equal risk. Let us however describe two cases in which specifying $\succsim^{*}$ is not required to obtain precise social welfare criteria.

The first case is the Bernoulli case in which $u_{i}=v_{i}$ for all $i \in N$. Then, by definition of $\mathcal{F}^{e}$, for all $f \in \mathcal{F}^{e}$ one always has $v_{i}(c e(f))=E\left(v_{i} \circ f\right)=E\left(v_{j} \circ f\right)=$ $v_{j}(c e(f))$ for all $i, j \in N$. This makes it unnecessary to seek an exact specification of

\footnotetext{
${ }^{9}$ Gajdos and Maurin (2004) actually characterize measures of inequality under risk and uncertainty that combine ex ante and ex post inequality. Our paper focuses on ex post inequality in order to satisfy Dominance.
} 
$\succsim^{*}$ since the common value $v_{i}(c e(f))$ provides a direct measure of social welfare (by weak monotonicity of $\left.\succsim^{*}\right)$. This case retrieves Theorem 1 in Fleurbaey (2010).

Although it has been adopted by most of the literature, the Bernoulli case is not uncontroversial. It implies relying on risk aversion to assess the allocation of resources, even when there is no risk. It is hard to see why we should rely on VNM functions to make interpersonal comparisons in allocations where risk is absent (see Chambers and Echenique, 2012). Our introductory example points out that the assumption may imply recommending transfers that increase resource inequality.

The fact that the $v_{i}$ functions may be different from the $u_{i}$ functions has actually been at the center of a discussion of the relevance of Harsanyi's theorem for utilitarianism. Sen (1976) and Weymark (1991) argued that, while Harsanyi's theorem establishes that social welfare is a sum of VNM utilities, it does not follow that it is the sum of individuals' well-being levels (which is the definition of utilitarianism), because the $v_{i}$ functions may be different from the $u_{i}$ functions. The dissent hence arises from the identification of individuals' VNM utility levels with their well-being levels. Sen (1976) and Weymark (1991) both contend that we would need separate arguments to justify this identification, but that there is no reason to believe it is true. ${ }^{10}$

The Bernoulli case is actually a particular case of a more general situation. This is when people have the same risk attitudes as described by functions $\phi_{i}$, but not necessarily risk neutrality. Recall that $u_{i}=\phi_{i} \circ v_{i}$. If for all $i \in N, \phi_{i}=\phi$, we obtain that for all $f \in \mathcal{F}, \omega \in \Omega$ and $i, j \in N, u_{i}\left(f^{e}(\omega)\right)=\phi \circ v_{i}\left(f^{e}(\omega)\right)=\phi \circ$ $v_{j}\left(f^{e}(\omega)\right)=u_{j}\left(f^{e}(\omega)\right)$. Hence, the same reasoning as above applies, since $v_{i}(c e(f))=$ $\phi^{-1}\left(E\left(u_{i} \circ f^{e}\right)\right)$ is equal among individuals.

Strengthening the Pareto principle makes it possible to obtain a more precise result while allowing for the possibility that $u_{i}$ and $v_{i}$ may be quite different, with heterogeneous risk attitudes.

Proposition 2 Under Assumptions 1, 2, 3 and 4, if the social ordering $R_{\mathcal{F}}$ satisfies Agreement, Dominance and Pareto for equal or no risk, then for all $f, g \in \mathcal{F}, f P_{\mathcal{F}} g$ whenever there exists $\varepsilon \in \mathbb{R}_{++}$such that

$$
\left(v_{i}\left(c e\left(f^{e}-\varepsilon\right)\right)\right)_{i \in N} R_{W}\left(v_{i}\left(c e\left(g^{e}+\varepsilon\right)\right)\right)_{i \in N} .
$$

\footnotetext{
${ }^{10}$ See Fleurbaey and Mongin (2016) for a recent analysis of this debate.
} 
Proof. See Appendix A.

Like before, we have the following Corollary (whose proof is similar to the proof of Corollary 1).

Corollary 2 Under Assumptions 1, 2, 3 and 4, if $R_{W}$ is continuous and the social ordering $R_{\mathcal{F}}$ satisfies Agreement, Dominance and Pareto for equal or no risk, then for all $f, g \in \mathcal{F}, f R_{\mathcal{F}} g$ if and only if

$$
c e\left(f^{e}\right) R_{X} c e\left(g^{e}\right)
$$

Like Corollary 1, Corollary 2 describes a three-step construction for ordering uncertain allocations. First, in each state of the world, we compute an EDE allocation. Then, we compute the certainty equivalent of this EDE allocation for all individuals. Last, we assess the distribution of the certainty equivalent using the social ordering for riskless allocations. Compared to Proposition 1 and Corollary 1, Proposition 2 and Corollary 2 thus give an exact description of the ordering $\succsim^{*}$, which corresponds to $R_{W}$.

\subsection{Rationality issues}

The criterion defined in Equation (2) satisfies not just Dominance but also first-order stochastic dominance. This property can be defined in the following way. For all $z \in X$ and $f \in \mathcal{F}$, let $\Pi_{z}(f)=p\left(\left\{\omega \in \Omega \mid z R_{X} f(\omega)\right\}\right)$ be the probability of states where $f$ is worse than the riskless allocation $z$. An act $f$ first-order stochastically dominates act $g$ if, for all $z \in X, \Pi_{z}(f) \leq \Pi_{z}(g)$. The social ordering $R_{\mathcal{F}}$ satisfies first-order stochastic dominance if, whenever act $f$ first-order stochastically dominates act $g$, it is the case that $f R_{\mathcal{F}} g$. Now, if $f$ first-order stochastically dominates $g$ it is necessarily the case that $f^{e}$ first-order stochastically dominates $g^{e}$, by Lemma 1 (iii). ${ }^{11}$ Expected utilities satisfy first-order stochastic dominance, thus if $f^{e}$ stochastically dominates $g^{e}$, then $E\left(u_{i} \circ\right.$ $\left.f^{e}\right) \geq E\left(u_{i} \circ g^{e}\right)$ for all $i \in N$. Given that $v_{i}\left(c e\left(f^{e}\right)\right)=\phi_{i}^{-1} \circ u_{i}\left(c e\left(f^{e}\right)\right)=\phi_{i}^{-1}\left(E\left(u_{i} \circ\right.\right.$ $\left.f^{e}\right)$ ), and that $\phi_{i}$ (and hence $\phi_{i}^{-1}$ ) is increasing, it must be the case that for every $i \in N, v_{i}\left(c e\left(f^{e}\right)\right) \geq v_{i}\left(c e\left(g^{e}\right)\right)$. By Assumption 2, it implies that $c e\left(f^{e}\right) R_{X} c e\left(g^{e}\right)$, and by Corollary 2 that $f R_{\mathcal{F}} g$.

\footnotetext{
${ }^{11}$ The criterion defined in Equation (2) satisfies continuity, so that we can invoke Lemma 1 (iii).
} 
However, the criterion defined in Equation (2) does not satisfy a stronger property of eventwise dominance. ${ }^{12}$ This is shown by the following example. Assume that $\Omega=\left\{\omega_{1}, \omega_{2}, \omega_{3}\right\}$, and $p\left(\omega_{1}\right)=p\left(\omega_{2}\right)=p\left(\omega_{3}\right)=1 / 3$. There are only two individuals: individual 1 is extremely risk averse whereas individual 2 is risk-neutral (with respect to well-being measured by $\left.v_{i}\right): E\left(u_{1} \circ f\right)=\min _{\omega \mid p(\omega)>0} v_{1}(f(\omega)) ; E\left(u_{2} \circ f\right)=E\left(v_{2} \circ f\right)$. Consider the egalitarian acts depicted in Table 1 (with $a \geq 5 / 3$ and $b \geq 0$ ).

\begin{tabular}{l|ccc|ccc} 
& \multicolumn{3}{|c|}{ Act $f$} & \multicolumn{3}{c}{ Act $g$} \\
\hline \hline States & $\omega_{1}$ & $\omega_{2}$ & $\omega_{3}$ & $\omega_{1}$ & $\omega_{2}$ & $\omega_{3}$ \\
\hline$v_{1}=v_{2}$ & 1 & 3 & $3 a-4$ & 0 & $b$ & $3 a-3$ \\
$v_{1}(c e(E D E))$ & \multicolumn{2}{|c|}{1} & \multicolumn{2}{|c}{0} \\
$v_{2}(c e(E D E))$ & \multicolumn{2}{|c|}{$a-1+b / 3$} \\
\multicolumn{2}{|c|}{ Conditional on $\left\{\omega_{1}, \omega_{2}\right\}$} \\
\hline$v_{1}(c e(E D E))$ & \multicolumn{3}{|c|}{0} \\
$v_{2}(c e(E D E))$ & \multicolumn{2}{|c|}{$b / 2$}
\end{tabular}

Table 1: Example of a violation of eventwise dominance

Conditionally on event $\left\{\omega_{3}\right\}$, act $f$ is obviously worse than act $g$. Conditionally on event $\left\{\omega_{1}, \omega_{2}\right\}$, act $f$ is also worse than act $g$, when inequality aversion is not too high and $b$ is large enough. But, for a given level of inequality aversion and a given $b$, act $f$ is unconditionally better than act $g$ for $a$ large enough, because the gain $b / 3-1$ for individual 2 becomes negligible when $a$ is great. One therefore obtains the paradox that $f$ is better than $g$ although it is worse conditionally on each event of a partition of $\Omega$. Such a situation is problematic in itself and generates problems in sequential decisions.

Indeed, let $\varepsilon \in \mathbb{R}_{++}$be small enough so that the preference for $f-\varepsilon$ over $g$ remains. Consider the following sequential decision problem. At stage 1 the decision maker can opt for $f-\varepsilon$ or delay the choice until stage 3. At stage 2, the decision maker learns

\footnotetext{
${ }^{12}$ Eventwise dominance is defined formally by invoking conditional orderings (Savage, 1972). Let $R_{\mathcal{F}}(q)$ denote the social ordering on $\mathcal{F}$ when the beliefs of the society (and the individuals) are represented by the probability measure $q$. Beliefs evolve when society learns that the true state is contained in the event $A \in \mathcal{A}$. The social ordering conditional on event $A \in \mathcal{A}$ is $R_{\mathcal{F}}\left(p_{A}\right)$, for $p_{A}$ defined as $p_{A}(\omega)=p(\omega) / p(A)$ if $\omega \in A$ and $p_{A}(\omega)=0$ otherwise. Eventwise dominance says that $f R_{\mathcal{F}} g$ whenever $f R_{\mathcal{F}}\left(p_{A}\right) g$ for all $A$ in a partition of $\Omega$. Note that individuals use expected utilities with updated beliefs to assess an act $f$ conditionally on $A$.
} 
if $\omega \in\left\{\omega_{1}, \omega_{2}\right\}$ or $\omega=\omega_{3}$. At stage 3 , the decision maker chooses between $f$ and $g$, if $f-\varepsilon$ was not chosen at stage 1 .

Note that in this sequence, it is possible to delay and secure the outcomes of $f$ whatever happens. As $f$ statewise dominates $f-\varepsilon$, it seems irrational to opt for $f-\varepsilon$ in stage 1 rather than waiting until stage 3 and choose $f$. But at stage 3 , we know that $g$ will appear better than $f$ conditionally on any piece of information that may be obtained in stage 2 . Therefore, time inconsistency is likely to occur here. A resolute decision-maker would moreover appear irrational because, for the criterion under consideration, there is every reason to use the information acquired in stage 2 when one is at stage 3 . Foreseeing that at stage 3 the choice will be for $g$, at stage 1 it therefore appears better to choose $f-\varepsilon$, which entails a quasi-violation of statewise dominance because it is possible to obtain $f$, which is better in every state of the world.

There is one way to avoid these difficulties: it consists in assuming that $R_{X}$ is a maximin criterion. In the above example, $f$ is then better than $g$ conditionally on event $\{1,2\}$, no matter how great $b$ is. It is clear that only an ordering giving absolute priority to the worst-off escapes the problem in this example. In the next section, we show that only maximin criteria can be consistently extended when Pareto for equal or no risk is combined with Expected utility at the social level (which guarantees that $R_{\mathcal{F}}$ satisfies eventwise dominance).

\section{Expected utility at the social level}

The rationality problems discussed in the previous section suggest that Dominance may be too weak a rationality principle. In this section, we examine the consequences of adopting a stronger rationality requirement, guaranteeing eventwise dominance.

\subsection{A Harsanyi-like theorem}

In the spirit of Harsanyi's approach, let us adopt the expected utility assumption for the social ordering $R_{\mathcal{F}}$. We rely here again on formal welfarism (Assumption 2) and formulate the assumption in terms of the VNM representation of $R_{W}$. Let $W \circ v \circ f$ denote the function

$$
W \circ v \circ f(\omega)=W\left(v_{1}(f(\omega)), \ldots, v_{n}(f(\omega))\right), \quad \forall \omega \in \Omega
$$

The following property is well defined when Assumption 3 is satisfied. 
Expected utility There is a continuous function $W$ on $V^{n}$ such that for all $f, g \in \mathcal{F}$, $f R_{\mathcal{F}} g$ if and only if $E(W \circ v \circ f) \geq E(W \circ v \circ g)$.

This property implies Dominance, because if $W \circ v \circ f(\omega) \geq W \circ v \circ g(\omega)$ for all $\omega \in \Omega$ we necessarily have $E(W \circ v \circ f) \geq E(W \circ v \circ g)$, and likewise for strict inequality. Note moreover that, under Assumption 2 and Agreement, for all $x, y \in X, x R_{\mathcal{F}} y$ if and only if $\left(v_{i}(x)\right)_{i \in N} R_{W}\left(v_{i}(x)\right)_{i \in N}$, while under Expected utility, this is also equivalent to $W\left(\left(v_{i}(x)\right)_{i \in N}\right) \geq W\left(\left(v_{i}(y)\right)_{i \in N}\right)$. This means that $W$ represents $R_{W}$ on $V^{n}$. Continuity of $W$ implies continuity of $R_{W}$. Such an assumption of continuity has been avoided in Proposition 1 to cover social preferences of the leximin type. Reciprocally, weak monotonicity of $R_{W}$ implies that $W$ is weakly monotonic.

To obtain sharp results, we will introduce the following condition on the probability measure $p$ and the measurable space $(\Omega, \mathcal{A})$.

Assumption 5 For any $A \in \mathcal{A}$ and $\kappa \in[0,1]$, there exists $A^{\prime} \in \mathcal{A}$ such that $A^{\prime} \subset A$ and $p\left(A^{\prime}\right)=\kappa p(A)$.

Assumption 5 is an assumption of Convex-rangedness. It guarantees that $\Omega$ can be partitioned in a subsets to obtain subjective probabilities that are as low as one wishes. The assumption is satisfied when $\Omega$ is an infinite set and $p$ is an atomless countably additive probability measure.

Imposing only Pareto for equal risk, one gets the following result.

Theorem 1 Under Assumptions 1, 2, 3 and 5, if the social ordering $R_{\mathcal{F}}$ satisfies Agreement, Pareto for equal risk and Expected utility, then there exists a vector $\left(\alpha_{1}, \cdots, \alpha_{n}\right) \in$ $\mathbb{R}_{+}^{n}$ such that for all $f, g \in \mathcal{F}, f R_{\mathcal{F}} g$ if and only

$$
\sum_{i \in N} \alpha_{i} E\left(u_{i} \circ f^{e}\right) \geq \sum_{i \in N} \alpha_{i} E\left(u_{i} \circ g^{e}\right)
$$

Proof. See Appendix A.

Theorem 1 suggests to derive the social welfare function used in the expected utility formula from a linear aggregation of individuals' VNM utility indices. The result is similar to Harsanyi's (1955) result, except that the formula is applied only to egalitarian acts (the EDE acts). 
Note also that Theorem 1 specifies Proposition 1 by providing a specific ordering $\succsim^{*}$ to aggregate well-being levels. Indeed, it can be written as implying that for all $f, g \in \mathcal{F}, f R_{\mathcal{F}} g$ if and only

$$
\sum_{i \in N} \alpha_{i} \phi_{i}\left(v_{i}\left(c e\left(f^{e}\right)\right)\right) \geq \sum_{i \in N} \alpha_{i} \phi_{i}\left(v_{i}\left(c e\left(g^{e}\right)\right)\right) .
$$

This makes clear that the aggregation of well-being from the certainty-equivalent allocation of the EDE depends on the $\phi_{i}$ functions, that embody individuals risk attitudes. However, in general, the formula does not impose constraints on what the social ordering $R_{X}$ should be. This will not be the case anymore when Pareto for equal risk is strengthened into Pareto for equal or no risk because then the $\phi_{i}$ functions will also have consequences for the social ranking of riskless allocations. This is examined in the next section.

\subsection{Pareto for equal and no risk and maximin criteria}

Although the class of criteria obtained in Theorem 1 is already quite precise, it does not single out specific weights $\left(\alpha_{i}\right)_{i \in N}$. In order to characterize a specific criterion, one could consider strengthening the Pareto for equal risk to Pareto for equal or no risk. We show that, if the ranking $R_{X}$ does not depend on risk preferences, we indeed obtain a precise description of the social ranking $R_{\mathcal{F}}$, but also a strong restriction on what $R_{X}$ can be - namely, it has to be a maximin criterion.

In order to introduce the condition that individual risk preferences do not influence the social ranking of riskless allocations, we need to be able to describe how the social ranking $R_{\mathcal{F}}$ changes when individual risk preferences change, and hence we have to introduce a multi-profile setting. Throughout this section we will retain the assumption that the individual preferences $R_{i}$ and the well-being indices $v_{i}$ on $X$ are fixed. However, we will allow risk attitudes, represented by the functions $\phi_{i}$ such that $u_{i}=\phi_{i} \circ v_{i}$, to vary from one profile to another. Hence a profile of preferences (given that preferences over riskless allocations are fixed) is described by the collection of functions $\Phi=\left(\phi_{1}, \cdots, \phi_{n}\right)$.

Social orderings may change depending on individual preferences and we denote $R_{\mathcal{F}}^{\Phi}$ and $R_{X}^{\Phi}$ the social ordering of acts and the social ordering of allocations for a given profile of preferences $\Phi$. We will reinterpret Assumption 2 as assuming that, for each profile $\Phi$, the social ordering $R_{X}^{\Phi}$ is based on the fixed well-being indices $v_{i}$, that 
are combined through an associated weakly monotonic ordering $R_{W}^{\Phi}$ (which may vary depending on $\Phi)$.

Let $V$ denote the range of function $v_{i}$ (the same for all individuals by Assumption 3 ). Let $C(V)$ be the set of continuous increasing functions from $V$ to $\mathbb{R}$. Any profile $\Phi$ must then belong to the set $C(V)^{n}$. But we focus our attention on the restricted domain $\mathcal{C} \subset C(V)^{n}$ of profiles such that one individual $i$ has a weakly greater risk aversion than everyone else, in the sense that for all $j \in N, \phi_{i}$ is at least as concave as $\phi_{j}$, i.e., there is a concave function $\varphi_{j}$ such that $\phi_{i}=\varphi_{j} \circ \phi_{j}$.

Let us now introduce the following property, according to which risk attitudes should not matter when risk is absent.

Invariance to risk attitudes for constant acts For all $\Phi, \Phi^{\prime} \in \mathcal{C}$, for all $x, y \in X$,

$$
x R_{\mathcal{F}}^{\Phi} y \Longleftrightarrow x R_{\mathcal{F}}^{\Phi^{\prime}} y
$$

This property is similar to a property with the same name in Chambers and Echenique (2012). As they explain, the property requires that in ranking profiles of riskless acts the social ordering should ignore risk attitudes. This property contrasts with the Bernoulli case in which VNM utilities are used as measures of individual welfare, even when there is no risk.

To obtain our next result, we require the social ordering for allocations to satisfy a basic transfer property. We assume that the social ordering $R_{X}$ is equitable in the sense that a more equal distribution of the well-being indices $v_{i}$ is weakly preferred. This corresponds to the Pigou-Dalton transfer principle applied directly to well-being levels.

Equity For all $\Phi \in \mathcal{C}$, for any $x, y \in X$, if there exist $\varepsilon \geq 0, i, j \in N$ such that:

1. $v_{k}(x)=v_{k}(y)$ for all $k \in N \backslash\{i, j\}$;

2. $v_{i}(y)+\varepsilon=v_{i}(x) \leq v_{j}(x)=v_{j}(y)-\varepsilon$;

then $x R_{\mathcal{F}}^{\Phi} y$.

Under this additional fairness requirement, we obtain the following result. 
Theorem 2 Under Assumptions 1, 2, 3, 4 and 5, if for all $\Phi \in \mathcal{C}$ the social ordering $R_{\mathcal{F}}^{\Phi}$ satisfies Agreement, Pareto for equal or no risk, Expected utility, Invariance to risk attitudes for constant acts, and Equity, then:

1. for all $x, y \in X$, for all $\Phi \in \mathcal{C}$ :

$$
x R_{\mathcal{F}}^{\Phi} y \Longleftrightarrow \min _{i} v_{i}(x) \geq \min _{i} v_{i}(y) .
$$

2. for all $f, g \in \mathcal{F}$, for all $\Phi \in \mathcal{C}$ :

$$
\begin{aligned}
f R_{\mathcal{F}}^{\Phi} g \Longleftrightarrow \min _{i} \phi_{i}^{-1}\left[\int_{\Omega} \phi_{i}\left(\min _{j} v_{j}(f(\omega))\right) d p(\omega)\right] \\
\geq \min _{i} \phi_{i}^{-1}\left[\int_{\Omega} \phi_{i}\left(\min _{j} v_{j}(g(\omega))\right) d p(\omega)\right] .
\end{aligned}
$$

Proof. Point 1 is obviously a corollary of point 2 , but it is convenient to prove it first. See Appendix A.

Observe that point 1 of Theorem 2 implies a very strong constraint on $R_{X}^{\Phi}$, namely that is based on a maximin social welfare ordering $R_{W}$ (since by Agreement, $R_{\mathcal{F}}^{\Phi}$ and $R_{X}^{\Phi}$ coincide on riskless allocations). Therefore, if the exogenous $R_{X}^{\Phi}$ is not a maximin, this theorem is an impossibility result. If infinite inequality aversion is considered ethically problematic, our paper offers two options: to accept weaker rationality requirements (Proposition 2) or a weaker Pareto principle (Theorem 1).

The criterion axiomatized in Theorem 2 is in stark contrast with the one proposed in Chambers and Echenique (2012, Example 10). They suggest that society should use the risk preferences of the least risk-averse individual (which is consistent with their axiom of reduction of risk aversion). Here we obtain that fairness and social rationality, on the contrary, require using the risk preferences of the most risk-averse individual. Indeed, for any $v: \mathcal{F} \rightarrow \mathbb{R}$, the minimal certainty-equivalent welfare $\min _{i} \phi_{i}^{-1}\left[\int_{\Omega} \phi_{i} \circ v(\omega) d p(\omega)\right]$ is obtained for the most concave function $\phi_{i}$ (which is always well-defined for profiles in $\mathcal{C}$ ), which corresponds to the most risk-averse person.

Theorem 2 is consistent with Theorem 1 that derives a weighted sum of expected utilities of the EDE. It amounts to picking a specific weighting scheme, where a positive weight is assigned to the most risk averse individual only, while all other agents have a zero weight. Note that the existence of a most risk averse agent is necessary to get the 
result: if the individual with the lower certainty-equivalent could change for different egalitarian acts, the weights could not be independent of the act, because the maximin would put all weight on a different individual depending on the act.

\subsection{Leximin social preferences}

One drawback of the maximin criterion is that it does not satisfy the Strong Pareto principle according to which a situation is strictly preferred to another whenever at least one individual strictly prefers the situation while no other individual weakly prefers the alternative. This may result in inefficient choices by a maximin criterion in some economic environments. As a consequence, the social choice literature typically suggests to use a leximin criterion rather than a maximin criterion.

In the context of the present article, the leximin criterion however raises two challenges. First, because it is not continuous, it does not provide an EDE, but only an EDQE, which is equal to the EDE of the maximin. Hence Proposition 2 does not completely specify the social ordering: cases with the same EDQE are not specified (while they are fully determined, in the continuous case, thanks to Corollary 2). Second, because the leximin criterion is not continuous, we cannot take its expectation to get an expected utility criterion at the social level.

If we are willing to use a more general setting than expected utility, we can design a version of leximin in the present setting. To describe this criterion let us introduce some more notation. For any $m \in \mathbb{N}$, and any $x=\left(x_{1}, \cdots, x_{m}\right), y=\left(y_{1}, \cdots, y_{m}\right) \in \mathbb{R}^{m}$, we write $x>_{\text {lex }}^{m} y$ if, after reordering the components of $x$ and $y$ in increasing order, there exists $k \in\{1, \cdots, m\}$ such that $x_{j}=y_{j}$ for all $j<k$ and $x_{k}>y_{k}$; we write $x \geq_{\text {lex }}^{m} y$ if either $x>_{\text {lex }}^{m} y$ or $x$ is a permutation of $y$. The leximin ordering $\geq_{\text {lex }}^{m}$ compares vectors of the same size $m \in \mathbb{N}$. In the remainder of this section, we focus on the case $m=n^{2}$, where $n \in \mathbb{N}$ is the population size.

For every $f \in \mathcal{F}$, let $\tilde{f} \in \mathcal{F}$ denote the act that reorders the well-being levels $v_{i}\left(f_{i}(\omega)\right)$ in increasing order for each $\omega \in \Omega$. This means that for all $\omega \in \Omega$ there exists a permutation $\pi: N \rightarrow N$ such that $v_{i}(\tilde{f}(\omega))=v_{\pi(i)}(f(\omega))$ for all $i \in N$, and for all $j, k \in N$,

$$
j<k \Longrightarrow v_{j}(\tilde{f}(\omega)) \leq v_{k}(\tilde{f}(\omega))
$$

Note that $\tilde{f}$ is not in general a permutation of $f$, even when we consider distributions 
of divisible goods in the population, since it permutes the utilities $v_{i}$, not the material payoffs. Hence act $\tilde{f}$ generates the same distribution of welfare as $f$ in every state of the world, but it creates fictional acts for individuals, where individual 1 always has the worst outcome in all states of the world, individual 2 has the second worst outcome, and so forth. Note that it is always possible to define $\tilde{f}$ under Assumption 3.

Next, for any $k \in N$, we define $f_{[k]}^{e} \in \mathcal{F}^{e}$ as the egalitarian act such that, for all $\omega \in \Omega$, for all $i \in N, v_{i}\left(f_{[k]}^{e}(\omega)\right)=v_{k}(\tilde{f}(\omega))$. It assigns an equal well-being level for all individuals, which is the well-being level of the $k$ th individual in terms of well-being with act $f$ in each state of the world. So it constructs a fictional situation where all individuals face a prospect that always gives them the $k$ th level of welfare.

Definition 2 The fair leximin ordering $R_{\mathcal{F}}^{l e x}$ is defined for any $f, g \in$ as,

$$
\begin{aligned}
f R_{\mathcal{F}}^{l e x} g \Longleftrightarrow & \left(\left(v_{i}\left(c e\left(f_{[1]}^{e}\right)\right)_{i \in N}, \cdots,\left(v_{i}\left(c e\left(f_{[n]}^{e}\right)\right)_{i \in N}\right)\right.\right. \\
& \geq_{l e x}^{n^{2}}\left(\left(v_{i}\left(c e\left(g_{[1]}^{e}\right)\right)_{i \in N}, \cdots,\left(v_{i}\left(c e\left(g_{[n]}^{e}\right)\right)_{i \in N}\right) .\right.\right.
\end{aligned}
$$

Note that when individuals have different risk attitudes, in general one does not have

$$
\max _{i \in N} v_{i}\left(c e\left(f_{[k]}^{e}\right)\right) \leq \min _{i \in N} v_{i}\left(c e\left(f_{[k+1]}^{e}\right)\right) .
$$

So, the leximin criterion proposed here may sometimes give the priority to a more risk-averse individual facing the better prospect $f_{[k+1]}^{e}$ over a less risk-averse individual facing a worse prospect $f_{[k]}^{e}$. This also explains why we have to use the ordering $\geq_{\text {lex }}^{n^{2}}$ of vectors in $\mathbb{R}^{n^{2}}$ rather than the usual vector inequality in the definition of our leximin criterion: the vectors $\left(\left(v_{i}\left(c e\left(f_{[1]}^{e}\right)\right)_{i \in N}, \cdots,\left(v_{i}\left(c e^{e}\left(f_{[n]}\right)\right)_{i \in N}\right)\right.\right.$ and $\left(\left(v_{i}\left(c e\left(g_{[1]}^{e}\right)\right)_{i \in N}, \cdots,\left(v_{i}\left(c e^{e}\left(g_{[n]}\right)\right)_{i \in N}\right)\right.\right.$ are not necessarily already ordered.

We do not seek to provide a complete characterization of the leximin criterion. But it is worth mentioning two properties satisfied by the criterion that can be used for such a characterization. We will just describe these properties informally, together with the reasoning that explains our definition of the leximin.

Step 1: Replace $f$ by the ordered $\tilde{f}$. By Dominance, $\tilde{f} I_{\mathcal{F}} f$.

Step 2: Create a $n$-replica of $N$, i.e., an economy with $n^{2}$ agents in which every $i$ is cloned to produce $n$ individuals identical to $i$ in the replica. An axiom of replication invariance would make the ordering over replica be the same as the ordering over original allocations. 
Step 3: In the replicated economy, for every $\omega$, give $\tilde{f}(\omega)$ to every $i$ of the original economy. Then consider every $k$ th rotation of $\left(v_{i}(\tilde{f}(\omega))\right)_{i \in N}$, involving a new allocation $\tilde{f}_{k}(\omega)$ defined by the condition $v_{k}\left(\tilde{f}_{k}(\omega)\right) \leq \ldots \leq v_{n}\left(\tilde{f}_{k}(\omega)\right) \leq v_{1}\left(\tilde{f}_{k}(\omega)\right) \leq$ $\ldots \leq v_{k-1}\left(\tilde{f}_{k}(\omega)\right)$, and give $\tilde{f}_{k}(\omega)$ to the $k$ th replicated population. By Dominance, this reordering does not alter the value of the allocation.

Step 4: The allocation constructed in Step 3 has the property that individuals' well-being levels are comonotonic (there is no reranking across states of nature), and that every type of risk attitude is submitted to every type of individual lottery. The Pareto principle restricted to situations in which this double property is satisfied would allow us to replace every individual lottery by its certainty-equivalent. This gives us the distribution of well-being depicted by the formula

$$
\left(\left(v_{i}\left(c e\left(f_{[1]}^{e}\right)\right)\right)_{i \in N}, \cdots,\left(v_{i}\left(c e\left(f_{[n]}^{e}\right)\right)\right)_{i \in N}\right) .
$$

This (riskless) distribution of well-being is not ordered, and therefore the leximin criterion must still be applied to this vector.

\section{$5 \quad$ Examples}

In this section we illustrate how our social orderings can be applied in two contexts: a macroeconomic risk affecting the marginal productivity of labor, and a catastrophic risk on future generations' consumption.

\subsection{Macroeconomic risk}

Drèze and Gollier (1993) and Gollier (1991) have studied the design of an unemployment insurance scheme in the presence of a macroeconomic risk.

Drèze and Gollier (1993) have argued in favor of indexing wages on GDP, so as to pool risk efficiently. Their line of argument focuses on efficiency. As they assume identical agents and as the consumption of all workers, whether employed or not, is equalized in every state, their argument would still be valid when using the certainty-equivalent of the EDE as a criterion. In a similar model, Gollier (1991) introduces heterogeneity of reservation wages among workers, and studies the optimal variation of unemployment allowances in the presence of shocks on productivity. Heterogeneity between agents 
makes the comparison with our approach more interesting and we therefore revisit his analysis.

Consider an economy composed of a unit mass of workers, each having a VNM utility function $\phi\left(c-m_{i} \ell\right)$, where $c \in \mathbb{R}_{+}$is consumption, $m_{i}$ is individual $i$ 's reservation wage, and $\ell=1$ when employed, 0 when unemployed. In any given state $\omega \in \Omega$, consumption is equal to $W(\omega)$ for the (employed) workers, with $W(\omega)$ the random wage rate; consumption is equal to an allowance $B(\omega)$ for the unemployed. Note that we assume that all individuals have the same risk preferences, described by $\phi$, with respect to "net-of-labor-disutility" consumption, $c-m_{i} \ell$.

The economy operates under the constraint that the expected profits of firms must be above a threshold (that describes profit opportunities abroad): ${ }^{13}$

$$
\int_{\Omega}(F(L(\omega), \omega)-W(\omega) L(\omega)-(1-L(\omega)) B(\omega)) d p(\omega) \geq \bar{v}
$$

where $F(L(\omega), \omega)$ is the (random) production function, $L(\omega)$ is total employment. The term $(1-L(\omega)) B(\omega)$ in the profit formula corresponds to the fact that risk-neutral firms have to pay taxes to fund unemployment allowances. We assume that investors are abstract foreign markets and we focus on the social welfare of the population of workers.

In any given state $\omega$, labor markets are perfectly competitive so that wages are determined by the spot-market equilibrium condition $F_{1}(L(\omega), \omega)=W(\omega)-B(\omega) .{ }^{14}$ Unemployment is hence voluntary and a worker is employed if $W(\omega)-m_{i} \geq B(\omega)$, so that total employment is determined by

$$
L(\omega)=G(W(\omega)-B(\omega))
$$

where $G$ is the cumulative distribution function of the parameter $m_{i}$.

The expected utility of an individual with reservation wage $m_{i}$ is equal to

$$
\int_{\Omega}\left[\phi\left(\max \left\{W(\omega)-m_{i}, B(\omega)\right\}\right)\right] d p(\omega)
$$

Gollier observes that the spot labor market achieves productive efficiency in every state, but full ex ante Pareto efficiency in risk sharing would require making

\footnotetext{
${ }^{13}$ Gollier (1991) also considers the case of risk-averse investors, but we focus here on the simpler case in which they are risk-neutral.

${ }^{14} F_{1}(L, \omega)$ denotes the derivative of $F$ with respect to $L$.
} 
$\max \left\{W(\omega)-m_{i}, B(\omega)\right\}$ a constant for every worker $i$, which is impossible with a uniform allowance $B(\omega)$. Gollier shows that a constrained efficient allowance program must make the following expression a constant across states of nature:

$$
\int_{0}^{W(\omega)-B(\omega)} \lambda(m) \phi^{\prime}(W(\omega)-m) d G(m)+\int_{W(\omega)-B(\omega)}^{+\infty} \lambda(m) \phi^{\prime}(B(\omega)) d G(m),
$$

where $\lambda(m)$ is some weight function reflecting an ethical prioritization of individuals with different values of the $m$ parameter. This is not surprising as ex ante Pareto efficiency corresponds to the maximization of some weighted utilitarian criterion. The consequence is that $B(\omega)$ will be countercyclical with respect to $W(\omega) .{ }^{15}$

Now consider the social ordering characterized in Theorem 1. Assume that individual well-being levels are measured by net-of-labor-disutility consumptions, so that individuals have the same risk attitude in $\phi$. The weights $\alpha_{i}$ in the representation of the social ordering presented in Theorem 1 do not matter, because all individuals have the same expected utility at EDE acts. This expected utility is the social welfare criterion. Suppose that the EDE net-of-labor-disutility consumption levels are given by the generalized mean formula:

$$
E D E(\omega)=\varphi^{-1}\left(\int_{0}^{W(\omega)-B(\omega)} \varphi(W(\omega)-m) d G(m)+\int_{W(\omega)-B(\omega)}^{+\infty} \varphi(B(\omega)) d G(m)\right),
$$

where $\varphi$ is an increasing and concave function.

The optimal policy solves the following program: $\max \int_{\omega} \phi[E D E(\omega)] d p(\omega)$ under the same profit constraint and the spot market equilibrium conditions as above. An analysis similar to the one in Gollier (1991) yields that the expression

$$
\frac{\phi^{\prime}[E D E(\omega)]}{\varphi^{\prime}[E D E(\omega)]}\left(\int_{0}^{W(\omega)-B(\omega)} \varphi^{\prime}(W(\omega)-m) d G(m)+\int_{W(\omega)-B(\omega)}^{+\infty} \varphi^{\prime}(B(\omega)) d G(m)\right)
$$

must be a constant across states of nature (see Appendix B for details).

When $\varphi=\phi$, one obtains a special case of formula (3) with $\lambda \equiv 1$, which is not surprising as the utility of the EDE is then equal to the utilitarian sum of utilities. When $\varphi$ is more concave than $\phi$, so that $\varphi=\chi \circ \phi$ for a concave transformation $\chi$, the expression becomes:

$$
\frac{\int_{0}^{W(\omega)-B(\omega)} \varphi^{\prime}(W(\omega)-m) d G(m)+\int_{W(\omega)-B(\omega)}^{+\infty} \varphi^{\prime}(B(\omega)) d G(m)}{\chi^{\prime} \circ \phi[E D E(\omega)]} .
$$

\footnotetext{
${ }^{15}$ The expression (3) is decreasing in $W(\omega)$ as well as in $B(\omega)$.
} 
In this formula both the numerator and the denominator decrease in $W(\omega)$ and $B(\omega)$, so that the ratio may depend on these two parameters in a different way and the counter-cyclicality result need not always hold. ${ }^{16}$

Interestingly, when the degree of priority of the worst-off goes to infinity, the EDE tends to $B(\omega)$ (assuming that unemployment is positive in every state $\omega$ ), so that the optimal policy is to have a constant unemployment allowance $B(\omega)$ (see Appendix B). The permanently unemployed, who are the worst-off in every state and get full priority, are fully insured, whereas those who work (at least in some states) are only partially insured. Note that this case is also a limit case of formula (3), when $\lambda(m)$ is positive only for the greatest values of $m$.

\subsection{Prevention of catastrophes}

Weitzman (2009) argued that it was hard to bound the amount of effort one should make for the future in the case of a possible future catastrophic climate change. Weitzman and the following literature (for a comprehensive discussion, see Millner, 2013) have focused on a riskless investment that transfers consumption to the future. Here we will examine the different problem of investing for the reduction of the probability of a catastrophe. ${ }^{17}$ Consider an economy with two generations, which is examining whether it is worth taxing the first generation in order to reduce the risk of a catastrophe for the second generation. One can think of the climate change threat, or the meteor threat, as concrete motivations for this example.

The population is $N=N_{1} \cup N_{2}$ (the two generations), and we assume that there is a single consumption good for simplicity. For simplicity, we also assume in this subsection that all individuals have the same preferences, represented by the VNM utility function $\phi$. Denote $n_{1}$ the number of people in generation $1, n_{2}$ the number of people in generation 2 , and $n=n_{1}+n_{2}$. The first generation's consumption is $\left(x_{i}\right)_{i \in N_{1}}$ and the second generation will have either $\left(c_{i}\right)_{i \in N_{2}}$ with probability $p$, which is a catastrophe, or $\left(y_{i}\right)_{i \in N_{2}}$ with probability $1-p$. A proportional tax on the first

\footnotetext{
${ }^{16}$ For instance, if $\varphi(x)=-x^{-1}, \chi(x)=x^{3} / 3$ (which is concave on $\mathbb{R}_{-}$), and $G$ the CDF of the lognormal distribution with mean 1 and standard deviation 2, the ratio increases with $W(\omega)$ and decreases with $B(\omega)$ around $(W(\omega), B(\omega))=(1, .2)$.

${ }^{17}$ Pindyck and Wang (2009) estimate what a representative-agent economy calibrated on the US would be willing to pay for such a policy.
} 
generation would reduce its consumption to $\left((1-\tau) x_{i}\right)_{i \in N_{1}}$, and the outcome will be a reduction in the probability $p$. How much of $\tau$ should one accept for a given reduction in $p$ ?

Consider a policy that raises taxes by a small amount $d \tau>0$ and decreases the probability by a small $d p<0$. To evaluate such a change, the Utilitarian approach uses the social welfare function ${ }^{18}$

$$
W(\tau, p)=\sum_{i \in N_{1}} \phi\left((1-\tau) x_{i}\right)+\sum_{i \in N_{2}}\left[p \phi\left(c_{i}\right)+(1-p) \phi\left(y_{i}\right)\right]
$$

Hence the change in social welfare induced by the policy $(d \tau, d p)$ is:

$$
\begin{aligned}
d W(\tau, p) & =-d \tau \sum_{i \in N_{1}} x_{i} \phi^{\prime}\left((1-\tau) x_{i}\right)-d p \sum_{i \in N_{2}}\left[\phi\left(y_{i}\right)-\phi\left(c_{i}\right)\right] \\
& =-\sum_{i \in N_{1}} x_{i} \phi^{\prime}\left((1-\tau) x_{i}\right) \times\left(d \tau+\frac{\sum_{i \in N_{2}}\left[\phi\left(y_{i}\right)-\phi\left(c_{i}\right)\right]}{\sum_{i \in N_{1}} x_{i} \phi^{\prime}\left((1-\tau) x_{i}\right)} d p\right) .
\end{aligned}
$$

We name 'marginal social willingness to tax' the quantity

$$
S W T=\frac{\sum_{i \in N_{2}}\left[\phi\left(y_{i}\right)-\phi\left(c_{i}\right)\right]}{\sum_{i \in N_{1}} x_{i} \phi^{\prime}\left((1-\tau) x_{i}\right)} .
$$

If $S W T>d \tau /(-d p)$, then the policy is (marginally) worth implementing from the social point of view. One sees that $S W T$ can be very sensitive to highly negative values taken by $\phi\left(c_{i}\right)$ when $c_{i}$ becomes small. The same is true in Weitzman's (2009) example, although it deals with investments that raise future consumption rather than reducing future risks.

Like in Section 5.1, let us study the implications of using the social ordering obtained in Theorem 1 and let us assume that $R_{X}$ is an inequality averse social ordering represented by the function $\sum_{i \in N} \varphi\left(x_{i}\right)$, with $\varphi$ a concave function. Let the EDE in the catastrophic and non-catastrophic cases be denoted

$$
\begin{aligned}
& E D E^{c}=\varphi^{-1}\left(\frac{1}{n}\left(\sum_{i \in N_{1}} \varphi\left((1-\tau) x_{i}\right)+\sum_{i \in N_{2}} \varphi\left(c_{i}\right)\right)\right), \\
& E D E^{y}=\varphi^{-1}\left(\frac{1}{n}\left(\sum_{i \in N_{1}} \varphi\left((1-\tau) x_{i}\right)+\sum_{i \in N_{2}} \varphi\left(y_{i}\right)\right)\right) .
\end{aligned}
$$

\footnotetext{
${ }^{18}$ We assume that the same weight is put on the utility function of all individuals, since there is no reason to treat differently people who have the same preferences.
} 
In the economy of this example, the social welfare criterion obtained in Theorem 1 takes the form ${ }^{19}$

$$
W^{*}(\tau, p)=p \phi\left(E D E^{c}\right)+(1-p) \phi\left(E D E^{y}\right) .
$$

Once again, we can compute a social willingness to tax:

$$
\begin{aligned}
S W T^{*}= & \frac{n\left[\phi\left(E D E^{y}\right)-\phi\left(E D E^{c}\right)\right]}{\sum_{i \in N_{1}} x_{i} \varphi^{\prime}\left((1-\tau) x_{i}\right)} \times \\
& \left(p \frac{\phi^{\prime}\left(E D E^{c}\right)}{\varphi^{\prime}\left(E D E^{c}\right)}+(1-p) \frac{\phi^{\prime}\left(E D E^{y}\right)}{\varphi^{\prime}\left(E D E^{y}\right)}\right)^{-1} .
\end{aligned}
$$

In this formula, the first ratio on the right-hand side is similar to the Utilitarian formula, with two key differences. First, the priority of individuals is determined by the $\varphi$ function and does not depend on risk attitudes. This can be defended as much preferable in terms of equity, because it provides more flexibility to determine social preferences for redistribution.

The second difference is that at the numerator all individuals are counted, not just the members of the second generation, because the benefit of reducing the risk of a catastrophe is shared by the whole society as a reduction in the probability of having the low $E D E^{c}$. In the social evaluation, the EDE approach implies that one must look at a situation in which all individuals suffer from risk, because if equality was achieved in all states, the risk borne by the second generation would carry over to the first one. Again, this is preferable (on egalitarian grounds) because it takes into account the degree of correlation of risk instead of adding up $\sum_{i \in N_{2}}\left(\phi\left(y_{i}\right)-\phi\left(c_{i}\right)\right)$ without checking whether the risk reduction is independent for every individual or involves a macroeconomic risk.

The final term on the right-hand side of the formula is a calibration term that takes account of how the ratio $\phi^{\prime} / \varphi^{\prime}$ varies across states of the world, and comes from the fact that the cost of the tax on the first generation is analyzed in terms of a cost on the EDE and is therefore shared by all individuals as well.

To compare the two approaches, let us focus on a simple case in which all individuals in a generation have identical consumption (either $x, y$ or $c$ depending on the generation

\footnotetext{
${ }^{19}$ Since individuals have the same utility function, the summation over all $i$, with weights $\alpha_{i}$, is not needed.
} 
and the state of the world). First assume that $\phi=\varphi$. Then the formula (5) simplifies into

$$
\begin{aligned}
S W T^{*} & =\frac{n\left[\phi\left(E D E^{y}\right)-\phi\left(E D E^{c}\right)\right]}{n_{1} x \varphi^{\prime}((1-\tau) x)} \\
& =\frac{n_{2}(\phi(y)-\phi(c))}{n_{1} x \phi^{\prime}((1-\tau) x)}
\end{aligned}
$$

which corresponds exactly to the utilitarian formula when people all have the same risk preferences.

Now assume that there exists an increasing function $\chi$ such that $\varphi=\chi \circ \phi$. One obtains (using the chain rule to obtain the derivative of function $\varphi$ )

$$
\begin{aligned}
S W T^{*}= & \frac{n\left(\phi\left(E D E^{y}\right)-\phi\left(E D E^{c}\right)\right)}{n_{1} x \phi^{\prime}((1-\tau) x) \times \chi^{\prime} \circ \phi((1-\tau) x)} \times \\
& \left(p \frac{\phi^{\prime}\left(E D E^{c}\right)}{\phi^{\prime}\left(E D E^{c}\right) \times \chi^{\prime} \circ \phi\left(E D E^{c}\right)}+(1-p) \frac{\phi^{\prime}\left(E D E^{y}\right)}{\phi^{\prime}\left(E D E^{y}\right) \times \chi^{\prime} \circ \phi\left(E D E^{y}\right)}\right)^{-1} . \\
= & \frac{n\left(\phi\left(E D E^{y}\right)-\phi\left(E D E^{c}\right)\right)}{n_{1} x \phi^{\prime}((1-\tau) x)} \times \\
& \left(p \frac{\chi^{\prime} \circ \phi((1-\tau) x)}{\chi^{\prime} \circ \phi\left(E D E^{c}\right)}+(1-p) \frac{\chi^{\prime} \circ \phi((1-\tau) x)}{\chi^{\prime} \circ \phi\left(E D E^{y}\right)}\right)^{-1} .
\end{aligned}
$$

Letting $S W T$ denote the utilitarian value

$$
S W T=\frac{n_{2}(\phi(y)-\phi(c))}{n_{1} x \phi^{\prime}((1-\tau) x)}
$$

one has $S W T^{*}=\varsigma \times S W T$, with

$\varsigma=\left(\frac{n\left(\phi\left(E D E^{y}\right)-\phi\left(E D E^{c}\right)\right)}{n_{2}(\phi(y)-\phi(c))}\right) /\left(p \frac{\chi^{\prime} \circ \phi((1-\tau) x)}{\chi^{\prime} \circ \phi\left(E D E^{c}\right)}+(1-p) \frac{\chi^{\prime} \circ \phi((1-\tau) x)}{\chi^{\prime} \circ \phi\left(E D E^{y}\right)}\right)$.

If $c \ll(1-\tau) x \simeq y$, then $\varsigma>1$ if $\chi$ is concave (inequality aversion greater than risk aversion). Indeed, one then has $E D E^{y} \simeq(1-\tau) x \simeq y$, and therefore:

$$
\varsigma \simeq\left(\frac{n\left(\phi(y)-\phi\left(E D E^{c}\right)\right)}{n_{2}(\phi(y)-\phi(c))}\right) /\left(p \frac{\chi^{\prime} \circ \phi(y)}{\chi^{\prime} \circ \phi\left(E D E^{c}\right)}+(1-p)\right) .
$$

By concavity of $\chi$ and $\phi\left(E D E^{c}\right)<\phi(y)$ (because $c \ll(1-\tau) x \simeq y$ and $\phi$ is increasing), the denominator is smaller than 1 . Moreover, the numerator is greater than 1 because

$$
\begin{aligned}
& \frac{n\left(\phi(y)-\phi\left(E D E^{c}\right)\right)}{n_{2}(\phi(y)-\phi(c))}>1 \\
\Leftrightarrow & \frac{n_{1}}{n} \phi(y)+\frac{n_{2}}{n} \phi(c)>\phi\left(E D E^{c}\right) \\
\Leftrightarrow & \phi^{-1}\left(\frac{n_{1}}{n} \phi(y)+\frac{n_{2}}{n} \phi(c)\right)>\varphi^{-1}\left(\frac{n_{1}}{n} \varphi((1-\tau) x)+\frac{n_{2}}{n} \varphi(c)\right) .
\end{aligned}
$$


The last inequality holds true when $\varphi$ is more concave than $\phi$ and $(1-\tau) x \leq y$.

Keeping $\chi$ concave, it is also possible to have $\varsigma<1$ when $c \simeq(1-\tau) x \ll y$. Indeed, one then has $E D E^{c} \simeq(1-\tau) x \simeq c$, and therefore:

$$
\varsigma \simeq\left(\frac{n\left(\phi\left(E D E^{y}\right)-\phi(c)\right)}{n_{2}(\phi(y)-\phi(c))}\right) /\left(p+(1-p) \frac{\chi^{\prime} \circ \phi(c)}{\chi^{\prime} \circ \phi\left(E D E^{y}\right)}\right),
$$

where the denominator is greater than 1 (by concavity of $\chi$ and $\phi\left(E D E^{y}\right)>\phi(c)$ ), whereas the numerator is smaller than 1 since

$$
\begin{aligned}
& \frac{n\left(\phi\left(E D E^{y}\right)-\phi(c)\right)}{n_{2}(\phi(y)-\phi(c))}<1 \\
\Leftrightarrow & \phi\left(E D E^{y}\right)<\frac{n_{1}}{n} \phi(c)+\frac{n_{2}}{n} \phi(y) \\
\Leftrightarrow & \varphi^{-1}\left(\frac{n_{1}}{n} \varphi((1-\tau) x)+\frac{n_{2}}{n} \varphi(y)\right)<\phi^{-1}\left(\frac{n_{1}}{n} \phi(c)+\frac{n_{2}}{n} \phi(y)\right) .
\end{aligned}
$$

Hence, when the future wellbeing level in the good state is similar to the current wellbeing level, and if inequality aversion is larger than risk aversion, our approach implies that the society is willing to pay more to reduce the risk of a catastrophe. On the contrary, when the catastrophe is not so bad (similar in terms of wellbeing to the current situation), our approach implies that the society is willing to pay less to reduce the risk of a catastrophe. The key intuition is fairness: special attention is given to the worst-off generation compared to the utilitarian formula.

As a further illustration, consider the case where $R_{X}$ is a maximin, which corresponds to $\chi$ being extremely concave. Assume that $c<(1-\tau) x<y$. In the maximin case, one has

$$
\begin{aligned}
& E D E^{c}=c, \\
& E D E^{y}=(1-\tau) x .
\end{aligned}
$$

The social welfare criterion obtained in Theorem 1 thus takes the form:

$$
W^{* *}(\tau, p)=p \phi(c)+(1-p) \phi((1-\tau) x),
$$

and the social willingness to tax is given by the formula:

$$
S W T^{* *}=\frac{\phi((1-\tau) x)-\phi(c)}{(1-p) x \phi^{\prime}((1-\tau) x)}=\varsigma^{*} \times S W T,
$$

with

$$
\varsigma^{*}=\frac{n_{1}(\phi((1-\tau) x)-\phi(c))}{n_{2}(\phi(y)-\phi(c))(1-p)} .
$$


In this case, the social willingness to tax, compared to the utilitarian formula, is diminished by the fact that the good state does not look so bright due to the lower welfare of the present generation, which reduces the comparative loss when a catastrophe occurs. The denominator now features the probability, and the willingness to tax now increases with the value of $p$, due to the fact that the cost of the tax on present generations is only counted in the good state.

\section{Uncertainty}

In this section we examine how to extend the analysis to the case of uncertainty, where individuals may have different beliefs, and perhaps even face ambiguity (represented by a set of probabilities). We first show that our main results extend to that case, so that dealing with uncertainty is possible within the general framework we have developed. We also study the case in which the decision criterion at the individual level is no longer expected utility but the maxmin expected utility. In a specific case, we show that social preferences may be maxmin expected utility, using a specific set of beliefs.

\subsection{The general result under uncertainty}

Up to now, we have assumed that acts can be ranked from individual $i$ 's point of view via an ordering $R_{i}$ over the set $\mathcal{F}$, which is represented by an expected utility. We do not make this assumption anymore, but we make the following assumption on $R_{i}{ }^{20}$

Assumption 6 For each $i \in N$, individual $i$ 's preferences $R_{i}$ are represented by a functional $U_{i}: \mathcal{F} \rightarrow \mathbb{R}$ that satisfies the following properties:

1. Dominance: If $U_{i}[f(\omega)] \geq U_{i}[g(\omega)]$ for all $\omega \in \Omega$, then $f R_{i} g$.

2. Certainty equivalence: For all $f \in \mathcal{F}$, there exists $c e(f) \in X$ such that $U_{i}[\operatorname{ce}(f)]=$ $U_{i}[f]$.

Conditions 1 and 2 in Assumption 6 imply that the range of the $U_{i}$ functional restricted to $X$ is the same as the range on $\mathcal{F}$. The first property embodies both the idea of Dominance and that of state independence. The second property guarantees

\footnotetext{
${ }^{20}$ In the statement of Dominance, $f(\omega), g(\omega) \in X$ refer to the constant act delivering $f(\omega)$ and $g(\omega)$ for sure. Thus the expression $U_{i}[f(\omega)]$ evaluates the constant act that yields the $f(\omega)$ in all states.
} 
the existence of certainty equivalent allocations that can be used to rank alternatives. It is satisfied by many models of choice under uncertainty and can be obtained by using a continuity property together with the other regularity conditions on preferences and on the set of allocations $X$ (see for instance Cerreia-Vioglio et al., 2011). We do not elaborate on that matter, given that this is not the core topic of the paper.

Recall that $Z=\left\{z \in \mathbb{R}^{n} \mid \exists f \in \mathcal{F}^{e}, z=\left(v_{i}(c e(f))\right)_{i \in N}\right\}$ is the set of individual welfare distributions that can be reached at certainty-equivalents of egalitarian acts.

\section{Proposition 3}

1. Under Assumptions 2, 3 and 6, if the social ordering $R_{\mathcal{F}}$ satisfies Agreement, Dominance and Pareto for equal risk, then there exists a weakly monotonic ordering ${ }^{*}$ on $Z$ such that for all $f, g \in \mathcal{F}, f P_{\mathcal{F}} g$ whenever there exists $\varepsilon \in \mathbb{R}_{++}$ such that

$$
\left(v_{i}\left(c e\left(f^{e}-\varepsilon\right)\right)\right)_{i \in N} \succsim^{*}\left(v_{i}\left(c e\left(g^{e}+\varepsilon\right)\right)\right)_{i \in N} .
$$

2. Under Assumptions 2, 3 and 6, if the social ordering $R_{\mathcal{F}}$ satisfies Agreement, Dominance and Pareto for equal or no risk, then for all $f, g \in \mathcal{F}, f P_{\mathcal{F}} g$ whenever there exists $\varepsilon \in \mathbb{R}_{++}$such that

$$
\left(v_{i}\left(c e\left(f^{e}-\varepsilon\right)\right)\right)_{i \in N} R_{W}\left(v_{i}\left(c e\left(g^{e}+\varepsilon\right)\right)\right)_{i \in N} .
$$

Proof. The proof is very similar to the one of Propositions 1 and 2. See Appendix A.

Proposition 3 is very general, for it allows a variety of normative views on the social aggregation of preferences to assess allocations and a variety of models of decisions under uncertainty for individuals. However, it does not deliver precise results about how attitudes towards uncertainty are aggregated. To obtain sharper results, we now turn to the special case of maxmin expected utilities.

\subsection{Maxmin expected utility}

When individuals are not able to form specific beliefs (in the sense of having a single probability measure), one may ask how a fair procedure should aggregate the perceived ambiguity. 
To investigate this issue, we will assume that individuals are maxmin expected utility maximizers (Gilboa and Schmeidler, 1989). We will assume that the set of states of the world can be written $\Omega=\Omega_{1} \times \Omega_{2}$, where $\Omega_{2}$ is a set whose $\sigma$-algebra $\mathcal{A}_{2}$ defines non-ambiguous events for which all individuals form the same beliefs represented by a common probability measure $p$ over the measurable space $\left(\Omega_{2}, \mathcal{A}_{2}\right)$. The nonambiguous events will play a role similar to that of roulette acts in an AnscombeAumann framework; they correspond to situations of "objective" risk where individuals may be expected utility maximizers (but it is objective only in the sense that all agents have the same beliefs represented by a common probability measure). Let $\overline{\mathcal{F}} \subset \mathcal{F}$ be the subset of acts $f$ such that for all $\omega_{2} \in \Omega_{2}$ and all $\omega_{1}, \omega_{1}^{\prime} \in \Omega_{1}, f\left(\omega_{1}, \omega_{2}\right)=f\left(\omega_{1}^{\prime}, \omega_{2}\right)$. These are unambiguous acts so that we are in a situation of objective risk.

Individuals perceive ambiguity with respect to events in the set $\Omega_{1}$ represented by elements of $\mathcal{A}_{1}$. This is formalized in the following way: agent $i$ holds beliefs represented by a (closed and convex) set $\mathcal{Q}_{i}$ of probabilities over the measurable space $\left(\Omega_{1}, \mathcal{A}_{1}\right)$.

For any $f \in \mathcal{F}$ and $q \in \mathcal{Q}_{i}$, let us define

$$
E_{q}\left(u_{i} \circ f\right)=\int_{\Omega_{1}}\left(\int_{\Omega_{2}} u_{i}\left(f\left(\omega_{1}, \omega_{2}\right)\right) d p\left(\omega_{2}\right)\right) d q\left(\omega_{1}\right) .
$$

Assumption 7 For each $i \in N$, there exists a utility function $u_{i}: X \rightarrow \mathbb{R}$ and a closed and convex set of $\mathcal{A}_{1}$-measurable probabilities on $\Omega_{1}, \mathcal{Q}_{i}$, such that, for all $f, g \in \mathcal{F}$, $f R_{i} g$ if and only if $\min _{q \in \mathcal{Q}_{i}} E_{q}\left(u_{i} \circ f\right) \geq \min _{q \in \mathcal{Q}_{i}} E_{q}\left(u_{i} \circ g\right)$.

The issue is to describe how our social orderings would aggregate perceived uncertainty, i.e., the sets $\mathcal{Q}_{i}$. This can be answered in a very clear way when we adopt a multi-profile setting and Pareto for equal or no risk, as we did to obtain Theorem 2.

We assume that individual preferences $R_{i}$ restricted to $X$ are fixed and represented by functions $v_{i}: X \rightarrow \mathbb{R}$. Individuals are maxmin expected utility maximizers, so that their preferences are completely characterized by their VNM utility function $u_{i}: X \rightarrow \mathbb{R}$ (which is ordinally equivalent to $v_{i}$, with a function $\phi_{i}$ satisfying $u_{i}=\phi_{i} \circ u_{i}$ ) and the set $\mathcal{Q}_{i}$. Note that on $\overline{\mathcal{F}}$ individuals are expected utility maximizers, and we will also assume that social preferences satisfy Expected utility on $\overline{\mathcal{F}}$.

A profile of uncertainty attitudes can thus be defined by the functions $\phi_{i}$ and the sets of beliefs $\mathcal{Q}_{i}$. Social orderings may change depending on individual uncertainty attitudes and we denote $R_{\mathcal{F}}^{\Phi}$ and $R_{X}^{\Phi}$ the social ordering of acts and the social ordering of allocations for a given profile of uncertainty attitudes $\Phi=\left(\left(\phi_{1}, \mathcal{Q}_{1}\right), \cdots,\left(\phi_{n}, \mathcal{Q}_{n}\right)\right)$. 
We will assume that, for each profile $\Phi$, the social ordering $R_{X}^{\Phi}$ satisfies Assumption 2, and that it is always based on fixed well-being indices $v_{i}$ (independent of $\Phi$ ) that are combined through a weakly monotonic social welfare ordering $R_{W}^{\Phi}$.

Let $V$ denote the range of $v_{i}$ and, as before, $C(V)$ be the set of continuous increasing functions from $V$ to $\mathbb{R}$. Let $\mathbf{P}$ denote the set of closed and convex sets of $\mathcal{A}_{1}$-measurable probabilities on $\Omega_{1}$. Any profile $\Phi$ must then belong to the set $(C(V) \times \mathbf{P})^{n}$. As before, we restrict attention to a domain $\mathcal{D} \subset(C(V) \times \mathbf{P})^{n}$ of profiles for which there exists a most risk averse function $\bar{\psi}$ (i.e., such that for all $i \in N$ there is a convex function $\varphi_{i}$ such that $\left.\phi_{i}=\varphi_{i} \circ \bar{\psi}\right)$ and an individual $j \in N$ such that $\left(u_{j}, \mathcal{Q}_{j}\right)=\left(\bar{\psi} \circ v_{i}, \mathcal{Q}_{i}\right)$ (i.e. an individual, who can be $i$ himself, with the most risk averse utility function and the same set of beliefs as $i$ ). As before, we introduce the requirement that uncertainty attitudes should not matter when uncertainty is absent.

Invariance to uncertainty attitudes for constant acts For all $\Phi, \Phi^{\prime} \in \mathcal{D}$, for all $x, y \in X$,

$$
x R_{X}^{\Phi} y \Longleftrightarrow x R_{X}^{\Phi^{\prime}} y .
$$

Proposition 4 Under Assumptions 2, 3, 5, 6 and 7 , if for all $\Phi \in \mathcal{D}$ :

(i) the social ordering $R_{X}^{\Phi}$ has a continuous associate $R_{W}^{\Phi}$;

(ii) each social ordering $R_{\mathcal{F}}^{\Phi}$ satisfies Agreement, Pareto for equal or no risk, Invariance to uncertainty attitudes for constant acts, Equity, and satisfies Expected utility on $\overline{\mathcal{F}}$;

then, for all $f, g \in \mathcal{F}$, for all $\Phi \in \mathcal{C}$ :

$$
\begin{aligned}
f R_{\mathcal{F}}^{\Phi} g \Longleftrightarrow \min _{q \in \cup_{i \in N}} \int_{\Omega_{1}}\left[\int_{\Omega_{2}} \bar{\psi}\left(\min _{j} v_{j}\left(f\left(\omega_{1}, \omega_{2}\right)\right)\right) d p\left(\omega_{2}\right)\right] d q\left(\omega_{1}\right) \\
\geq \min _{q \in \cup_{i \in N}} \int_{\Omega_{1}}\left[\int_{\Omega_{2}} \bar{\psi}\left(\min _{j} v_{j}\left(g\left(\omega_{1}, \omega_{2}\right)\right)\right) d p\left(\omega_{2}\right)\right] d q\left(\omega_{1}\right) .
\end{aligned}
$$

Proof. The proof combines the results of Theorem 2 and Proposition 3. Details can be found in Appendix A. Note that Assumption 6 is still needed here to ensure the existence of the certainty equivalent, which is not guaranteed by Assumption 7 in our general framework.

Hence, we obtain that social ambiguity is strictly larger than individual ambiguity, because we have to take into account all the possible beliefs people in the society may 
hold. The social preferences for redistribution, embodied in the $R_{X}$ ordering, determine how ambiguity is aggregated. This explains why a preference for the worst-off may imply a focus on the worst possible probability held by people in the society. ${ }^{21}$

\section{Conclusion and discussion}

In this paper we have offered a methodology for the extension of a social ordering initially defined on riskless allocations to the set of risky allocations, and even to the context of uncertain allocations with ambiguity. The principles of Pareto for equal risk and Pareto for equal or no risk have played a central role in this analysis, making it possible, in combination with Dominance, to evaluate allocations in terms of the distribution of the certainty-equivalent, among individuals, of the equally-distributed equivalent.

In the context of risk (i.e., unambiguous and common probabilities), strengthening Dominance to Expected utility, which seems required for rationality purposes, yields an affine aggregation of risk attitudes when we assume the weak Pareto for equal risk principle. If we want to further strengthen Pareto (Pareto for equal or no risk), it appears impossible to satisfy Expected utility under Equity, unless the social ordering $R_{X}$ depends on risk attitudes, or is of the maximin kind. This suggests that it may be difficult to go beyond Pareto for equal risk when preferences are heterogenous, unless we endorse specific views on how wellbeing is measured.

In the context of uncertainty (ambiguous probabilities), the results extend quite smoothly, because what is needed for the main arguments is the existence of certainty equivalent alternatives, not that individuals satisfy the expected utility assumptions. If they follow the maxmin expected utility rule, as studied in the last section, and if social preferences satisfy Pareto for equal or no risk and the expected utility property on unambiguous acts, then, under some assumptions, social preferences are also of the

\footnotetext{
${ }^{21}$ Danan et al. (2016) obtain a seemingly opposite result, where social evaluation is made with the intersection of individual belief sets $\mathcal{Q}_{i}$ instead of the union. They rely on a different Pareto condition, which is not limited when risk generates inequalities, but is restricted to cases in which individual preferences are robust to the selection of beliefs in $\mathcal{Q}_{i}$. Their result bears only on the robust part of social preferences, and is compatible with idiosyncratic beliefs playing a role in the non-robust part. Furthermore, when they further restrict Pareto to acts on which individual VNM functions are identical up to an affine transform, which is in the spirit of Pareto for equal or no risk, the union of individual sets emerges in their result.
} 
maxmin expected utility sort and exhibit extreme ambiguity aversion in the sense of taking the most pessimistic of the whole set of population beliefs.

Incorporating ambiguity aversion into social preferences may, however, be questionable, as there is a debate about the normative appeal of ambiguity aversion in terms of rationality (Al-Najjar and Weinstein, 2009; Heal and Millner, 2015; Gilboa, 2015). The irrationality phenomena (violation of eventwise dominance, time inconsistency, refusal of free information), discussed in Section 3.2 to justify the Expected utility principle, appear again when social preferences exhibit ambiguity aversion.

Another problem when individuals have heterogenous beliefs is that unanimity may lose its normative appeal (Mongin, 2016). If individuals have different beliefs, they may agree to deem an option better than another one simply because they disagree on the prospects of the different outcomes being realized. For instance a very risk averse individual and a risk neutral individual may agree that an uncertain level of a public good is better than a sure level of public good only because the former believes that there is a very small probability that the level of public good will be very low, while the second believes that the expected level of public good is higher than the sure level of public good, but only slightly so. If the first was to realize that he was underestimating the probability of the bad outcome, he would not want to follow the uncertain course of action. This issue of spurious unanimities has been investigated within the framework of the expected utility model by several authors, including Gilboa et al. (2004). ${ }^{22}$

Both sets of issues can be avoided by separating the aggregation of beliefs from the aggregation of preferences. ${ }^{23}$ Such a separation means that the Pareto principle is then applied only with both individual and social prospects being evaluated with the same "aggregate" beliefs. Under this separation approach, the first part of our paper can be interpreted as applying to the aggregation of preferences, with the $p$ probability

\footnotetext{
${ }^{22}$ Note that the aggregation of preferences in our paper avoids the strongest form of spurious unanimities in which people have different beliefs and different rankings of final consequences in states of the world. The last feature of spurious unanimities is removed by focusing on egalitarian acts.

${ }^{23}$ Most of the literature in economics about social choice under uncertainty has explored the joint aggregation of tastes and beliefs, following the seminal contribution by Gilboa et al. (2004). Recent contributions include but are not limited to Chambers and Hayashi (2006), Nascimento (2012), and Danan et al. (2016). Some authors argue that it may be difficult to perform such a joint aggregation, in particular because it is not simple to identify the source of belief divergence from individual choices (see for instance Mongin and Pivato, 2016).
} 
measure embodying the aggregate beliefs.

To conclude, let us briefly mention open questions that deserve future attention. We have adopted a rational approach (Dominance, Expected utility) at the cost of ignoring ex ante fairness, since the way in which risky allocations shape the distribution of ex ante prospects is lost when an ex post allocation is replaced by its equally-distributed equivalent by the social preferences studied in this paper. Combining rationality and a concern for ex ante fairness remains largely an open question (see Gajdos and Maurin, 2004; Fleurbaey et al., 2015, for some explorations). Another set of open questions has to do with changes in the composition of the population depending on the state of nature that is realized, or depending on the allocation that is chosen. This brings in new issues about the evaluation of population size, and risks of changes in the population preferences, as discussed in the literature on climate policy (see Asheim and Zuber, 2016; Fleurbaey and Zuber, 2016, for forays into this field).

\section{Appendix A: Proofs of the main results}

\section{Proof of Lemma 1}

Suppose that Assumption 2 and 3 hold.

Proof of (i). Consider $x \in X$. By Assumption 3, there are $y, y^{\prime} \in X^{e}$ such that $v_{i}\left(y_{i}\right)=$ $\min _{j \in N} v_{j}\left(x_{j}\right)$ and $v_{i}\left(y_{i}^{\prime}\right)=\max _{j \in N} v_{j}\left(x_{j}\right)$ for all $i \in N$. By weak monotonicity of $R_{W}$ (Assumption 2), one has $y^{\prime} R_{X} x R_{X} y$.

Let $W=\left\{z \in X^{e} \mid x R_{X} z\right\}$ and $B=\left\{z \in X^{e} \mid z R_{X} x\right\}$. Since $y \in W$ and $y^{\prime} \in$ $B$, these two sets are non-empty. By transitivity, for all $\left(z, z^{\prime}\right) \in B \times W$, one has $z R_{X} x R_{X} z^{\prime}$, therefore $z R_{X} z^{\prime}$, and by weak monotonicity, for all $i \in N, v_{i}(z) \geq v_{i}\left(z^{\prime}\right)$. Therefore, for every $i, \sup v_{i}(W) \leq \inf v_{i}(B)$.

Suppose that $\sup v_{i}(W)<\inf v_{i}(B)$ (for any $i$-remember that $W$ and $B$ contain only egalitarian allocations). By Assumption 3, there is $z^{*} \in X^{e} \operatorname{such}$ that $\sup v_{i}(W)<$ $v_{i}\left(z^{*}\right)<\inf v_{i}(B)$. Necessarily $z \notin W \cup B$. But this is impossible, because it implies $z P_{X} x$ (since $\left.z \notin W\right)$ and $x P_{X} z($ since $z \notin B)$. Therefore $\sup v_{i}(W)=\inf v_{i}(B)$. By Assumption 3, there is $z^{*} \in X^{e}$ such that $\sup v_{i}(W)=v_{i}\left(z^{*}\right)=\inf v_{i}(B)$.

Let $z \in D\left(z^{*}\right)$. Assume that $x R_{X} z$. By Assumption 3, there is $z^{\prime} \in X^{e} \cap D\left(z^{*}\right)$ such that $v_{i}\left(z_{i}^{\prime}\right)=\min _{j \in N} v_{j}\left(z_{j}\right)>v_{i}\left(z_{i}^{*}\right)$ for all $i$. By weak monotonicity, $z R_{X} z^{\prime}$, implying by transitivity that $x R_{X} z^{\prime}$, so that $z^{\prime} \in W$. But that is impossible because 
$v_{i}\left(z_{i}^{\prime}\right)>\sup v_{i}(W)$. Therefore one must have $z P_{X} x$.

Similarly, one shows that if $z^{*} \in D(z)$ for some $z$, then one must have $x P_{X} z$. This shows that $z^{*}$ is an EDQE for $x$.

Let $z^{* *}$ be another EDQE of $x$. The proof that $z^{* *} I_{X} z^{*}$ is a direct corollary of (ii). Proof of (ii). Let $x^{e} P_{X} y^{e}$. Note that by weak monotonicity and Assumption 3, there is $z \in X^{e}$ such that $x^{e} P_{X} z P_{X} y^{e}$. One has $z \in D\left(y^{e}\right)$ and $x^{e} \in D(z)$, implying that $z P_{X} y$ and $x P_{X} z$. By transitivity, the result follows.

Proof of (iii). Assume that $x^{e} P_{X} x$. Then by continuity of $R_{W}$, there is $\varepsilon>0$ such that $v_{i}\left(x^{e}\right)-\varepsilon \in V$ and $\left(v_{i}\left(x^{e}\right)-\varepsilon\right)_{i \in N} P_{W}\left(v_{i}(x)\right)_{i \in N}$. This means that there is $z \in X^{e}$ such that $x^{e} P_{X} z P_{X} x$, which contradicts the fact that since $x^{e} \in D(z)$, one should have $x P_{X} z$ by definition of the EDQE. A similar contradiction arises if $x P_{X} x^{e}$.

\section{Proof of Proposition 1}

Suppose that Assumptions 1, 2, 3 and 4 hold and that the social ordering $R_{\mathcal{F}}$ satisfies Agreement, Dominance and Pareto for equal risk.

By definition of the certainty-equivalent, we have, for all $f \in \mathcal{F}, u_{i}(c e(f))=E\left(u_{i}\right.$ 。 $f$ ) so that $v_{i}(c e(f))=\phi_{i}^{-1}\left(E\left(u_{i} \circ f\right)\right.$ ) and, for all $f, g \in \mathcal{F}$, and for all $i \in N$ (by Assumption 1):

$$
f R_{i} g \Longleftrightarrow E\left(u_{i} \circ f\right) \geq E\left(u_{i} \circ g\right) \Longleftrightarrow v_{i}(c e(f)) \geq v_{i}(c e(g))
$$

Step 1. Definition and properties of $\succsim^{*}$.

Recall that $Z=\left\{z \in \mathbb{R}^{n} \mid \exists f \in \mathcal{F}^{e}, z=\left(v_{i}(c e(f))\right)_{i \in N}\right\}$. Define the binary relation $\succsim^{*}$ on $Z$ by the following condition: $z \succsim^{*} z^{\prime}$ if there exists $f, g \in \mathcal{F}^{e}$ such that $z=\left(v_{i}(c e(f))\right)_{i \in N}, z^{\prime}=\left(v_{i}(c e(g))\right)_{i \in N}$ and $f R_{\mathcal{F}} g$.

The relation $\succsim^{*}$ is an ordering on $Z$, because $R_{\mathcal{F}}$ is an ordering, and for each $z \in Z$ there exists $f \in \mathcal{F}^{e}$ such that $z=\left(v_{i}(c e(f))\right)_{i \in N}$. The relation $\succsim^{*}$ is also weakly monotonic. For $z, z^{\prime} \in Z$ such that $z \geq z^{\prime}$, there exist $f, g \in \mathcal{F}^{e}$ such that $z=\left(v_{i}(c e(f))\right)_{i \in N}, z^{\prime}=\left(v_{i}(c e(g))\right)_{i \in N}$ and $v_{i}(c e(f)) \geq v_{i}(c e(g))$ for all $i \in N$, so that, by Pareto for equal risk, $f R_{\mathcal{F}} g$, and by definition $z \succsim^{*} z^{\prime}$. A similar reasoning holds to show that for any $z, z^{\prime} \in Z$, if $z \gg z^{\prime}$ then $z \succ^{*} z^{\prime}$.

By Pareto for equal risk, if the egalitarian prospects $f, f^{\prime} \in \mathcal{F}^{e}$ are such that $\left(v_{i}(c e(f))\right)_{i \in N}=\left(v_{i}\left(c e\left(f^{\prime}\right)\right)\right)_{i \in N}=z$, then $f I_{\mathcal{F}} f^{\prime}$. So, by definition of $\succsim^{*}$, for all 
$f, g \in \mathcal{F}^{e}$

$$
\left(v_{i}(c e(f))\right)_{i \in N} \succsim^{*}\left(v_{i}(c e(g))\right)_{i \in N} \Longleftrightarrow f R_{\mathcal{F}} g .
$$

Step 2. Proof that $f P_{\mathcal{F}} g$ whenever $f^{e}$ dominates $g^{e}$.

Let $f, g \in \mathcal{F}$ be such that for all $\omega \in \Omega$ and for all $i \in N, v_{i}\left(f^{e}(\omega)\right)>v_{i}\left(g_{i}^{e}(\omega)\right)$. By Assumption 2, this implies that $f^{e}(\omega) P_{X} g^{e}(\omega)$. By Lemma 1 (ii), this implies $f(\omega) P_{X} g(\omega)$. Since this is true for all $\omega \in \Omega$, Dominance and Agreement imply that $f P_{\mathcal{F}} g$.

Step 3. Proof of the proposition.

Let $f, g \in \mathcal{F}$, be such that there exists $\varepsilon \in \mathbb{R}_{++}$such that

$$
\left(v_{i}\left(c e\left(f^{e}-\varepsilon\right)\right)\right)_{i \in N} \succsim^{*}\left(v_{i}\left(c e\left(g^{e}+\varepsilon\right)\right)\right)_{i \in N} .
$$

Let $f^{\prime}$ be the act $f^{e}-\varepsilon$ and $g^{\prime}$ be the act $g^{e}+\varepsilon$. By Step 1, given that $f^{\prime}, g^{\prime} \in \mathcal{F}^{e}$,

$$
\left(v_{i}\left(c e\left(f^{\prime}\right)\right)\right)_{i \in N} \succsim^{*}\left(v_{i}\left(c e\left(g^{\prime}\right)\right)\right)_{i \in N}
$$

implies that $f^{\prime} R_{\mathcal{F}} g^{\prime}$.

By Step 2, because $v_{i}\left(f^{e}(\omega)\right)>v_{i}\left(f^{\prime}(\omega)\right)$ for all $i \in N$ and all $\omega \in \Omega$, then $f P_{\mathcal{F}} f^{\prime}$ $\left(f^{\prime}\right.$ is an egalitarian act and thus its own EDQE act). Because $v_{i}\left(g^{e}(\omega)\right)<v_{i}\left(g^{\prime}(\omega)\right)$ for all $i \in N$ and all $\omega \in \Omega$, then $g^{\prime} P_{\mathcal{F}} g$.

By transitivity of $R_{\mathcal{F}}$, we thus obtain $f P_{\mathcal{F}} g$.

\section{Proof of Corollary 1}

Suppose that Assumptions 1, 2, 3 and 4 hold, that $R_{W}$ is continuous, and that the social ordering $R_{\mathcal{F}}$ satisfies Agreement, Dominance and Pareto for equal risk.

By Lemma 1 (iii), given that $R_{W}$ is continuous, then for all $x \in X, x^{e} I_{X} x$. Thus for all $f, g \in \mathcal{F}, f(\omega) I_{X} f^{e}(\omega)$ and $g(\omega) I_{X} g^{e}(\omega)$ for all $\omega \in \Omega$. By Agreement and Dominance, we then have $f I_{\mathcal{F}} f^{e}$ and $g I_{\mathcal{F}} g^{e}$ so that $f R_{\mathcal{F}} g \Longleftrightarrow f^{e} R_{\mathcal{F}} g^{e}$.

We can proceed like in Step 1 of the proof of Proposition 1 to show that there exists a complete and weakly monotonic relation $\succsim^{*}$ on $Z$ such for all $f, g \in \mathcal{F}^{e}$ :

$$
\left(v_{i}(c e(f))\right)_{i \in N} \succsim^{*}\left(v_{i}(c e(g))\right)_{i \in N} \Longleftrightarrow f R_{\mathcal{F}} g .
$$

Thus

$$
\begin{aligned}
f R_{\mathcal{F}} g & \Longleftrightarrow f^{e} R_{\mathcal{F}} g^{e} \\
& \Longleftrightarrow\left(v_{i}\left(c e\left(f^{e}\right)\right)\right)_{i \in N} \succsim^{*}\left(v_{i}\left(c e\left(g^{e}\right)\right)\right)_{i \in N} .
\end{aligned}
$$




\section{Proof of Proposition 2}

Suppose that Assumptions 1, 2, 3 and 4 hold, and that the social ordering $R_{\mathcal{F}}$ satisfies Agreement, Dominance and Pareto for equal or no risk.

Assume that there exists $\varepsilon \in \mathbb{R}_{++}$such that

$$
\left(v_{i}\left(c e\left(f^{e}-\varepsilon\right)\right)\right)_{i \in N} R_{W}\left(v_{i}\left(c e\left(g^{e}+\varepsilon\right)\right)\right)_{i \in N} .
$$

Let $f^{\prime}$ be the act $f^{e}-\varepsilon$ and $g^{\prime}$ be the act $g^{e}+\varepsilon$. By definition, $f^{\prime}, g^{\prime} \in \mathcal{F}^{e}$ and, for all $i \in N, c e\left(f^{\prime}\right) I_{i} f^{\prime}$ and $c e\left(g^{\prime}\right) I_{i} g^{\prime}$. By Pareto for equal or no risk, $c e\left(f^{\prime}\right) I_{\mathcal{F}} f^{\prime}$ and $c e\left(g^{\prime}\right) I_{\mathcal{F}} g^{\prime}$. By Assumption 2 and Agreement, because $c e\left(f^{\prime}\right), c e\left(g^{\prime}\right) \in X$,

$$
\left(v_{i}\left(c e\left(f^{\prime}\right)\right)\right)_{i \in N} R_{W}\left(v_{i}\left(c e\left(g^{\prime}\right)\right)\right)_{i \in N} .
$$

is equivalent to $c e\left(f^{\prime}\right) R_{\mathcal{F}} c e\left(g^{\prime}\right)$. By transitivity, $f^{\prime} R_{\mathcal{F}} g^{\prime}$.

By Step 2 of the proof of Proposition 1, because $v_{i}\left(f^{e}(\omega)\right)>v_{i}\left(f^{\prime}(\omega)\right)=v_{i}\left(f^{\prime e}(\omega)\right)$ for all $i \in N$ and all $\omega \in \Omega$, then $f P_{\mathcal{F}} f^{\prime}$, and because $v_{i}\left(g^{e}(\omega)\right)<v_{i}\left(g^{\prime}(\omega)\right)=v_{i}\left(g^{\prime e}(\omega)\right)$ for all $i \in N$ and all $\omega \in \Omega$, then $g^{\prime} P_{\mathcal{F}} g$.

By transitivity of $R_{\mathcal{F}}$, we thus obtain that $f P_{\mathcal{F}} g$.

\section{Proof of Theorem 1}

Suppose that Assumptions 1, 2, 3 and 5 hold, and that the social ordering $R_{\mathcal{F}}$ satisfies Agreement, Pareto for equal risk and Expected utility.

By Expected utility, $R_{\mathcal{F}}$ satisfies Dominance and $R_{W}$ is continuous. By Lemma 1 (iii), for all $f, g \in \mathcal{F}, f(\omega) I_{X} f^{e}(\omega)$ and $g(\omega) I_{X} g^{e}(\omega)$ for all $\omega \in \Omega$. By Agreement and Expected utility (Dominance), we then have $f I_{\mathcal{F}} f^{e}$ and $g I_{\mathcal{F}} g^{e}$ so that $f R_{\mathcal{F}} g \Longleftrightarrow$ $f^{e} R_{\mathcal{F}} g^{e}$.

By Expected utility, we also know that there exists a function $W$ such that for all $f, g \in \mathcal{F}:$

$$
f R_{\mathcal{F}} g \Longleftrightarrow E(W \circ v \circ f) \geq E(W \circ v \circ g)
$$

By Assumption 2 and Agreement, $W$ must be weakly monotonic.

Define $\mathcal{L}^{e}$ the set of finite lotteries over the set $X^{e}$, i.e., the set of functions $q: X^{e} \rightarrow$ $[0,1]$ such that $\sum_{x \in X^{e}} q(x)=1$ and $q(x)>0$ for a finite number of elements $x \in X^{e}$. $\mathcal{L}^{e}$ is a mixture set: for any $q, q^{\prime} \in \mathcal{L}^{e}$ and $\lambda \in[0,1]$, the lottery $\hat{q}=\lambda q+(1-\lambda) q^{\prime}$, defined by $\hat{q}(x)=\lambda q(x)+(1-\lambda) q^{\prime}(x)$ for all $x \in X^{e}$, belongs to $\mathcal{L}^{e}$. 
To every $q \in \mathcal{L}^{e}$, we can associate an act $f_{q} \in \mathcal{F}^{e}$ in the following way. Let $\left\{x_{1}, \cdots, x_{k}\right\}$ be the finite set of elements of $X^{e}$ such that $q\left(x_{l}\right)>0$, where $l \in\{1, \cdots, k\}$. By iterative application of Assumption 5, it is possible to find a partition $\left\{A_{1}, \cdots, A_{k}\right\}$ of $\Omega$ such that $p\left(A_{l}\right)=q\left(x_{l}\right)$ for all $l \in\{1, \cdots, k\}$. We define $f_{q}$ by $f_{q}(\omega)=x_{l}$ for all $\omega \in A_{l}$, for all $l \in\{1, \cdots, k\}$.

Similarly, to every $f \in \mathcal{F}^{e}$ we can associate a lottery $q_{f} \in \mathcal{L}^{e}$ in the following way. Given that $f$ is a simple act, there exists a finite a partition $\left\{A_{1}, \cdots, A_{k}\right\}$ of $\Omega$ and a finite finite set $\left\{x_{1}, \cdots, x_{k}\right\} \subset X^{e}$, such that $f(\omega)=x_{l}$ for all $\omega \in A_{l}$, $l \in\{1, \cdots, k\}$. We define $q_{f}$ as the function $q_{f}: X^{e} \rightarrow[0,1]$ such that $q_{f}\left(x_{l}\right)=p\left(A_{l}\right)$ for all $l \in\{1, \cdots, k\}$ and $q_{f}(x)=0$ for all $x \in X^{e} \backslash\left\{x_{1}, \cdots, x_{k}\right\}$. It is straightforward that $q_{f} \in \mathcal{L}^{e}$.

We can thus define $V_{i}(q)=\sum_{x \in X^{e}} q(x) u_{i}(x)=E\left(u_{i} \circ f_{q}\right)$ and $V_{0}(q)=\sum_{x \in X^{e}} q(x) W \circ$ $v(x)=E\left(W \circ v \circ f_{q}\right)$. For all $q, q^{\prime} \in \mathcal{L}^{e}$ and $\lambda \in[0,1]$, and all $j \in\{0,1, \cdots, n\}$, we have $V_{j}\left(\lambda q+(1-\lambda) q^{\prime}\right)=\lambda V_{j}(q)+(1-\lambda) V\left(q^{\prime}\right)$. Hence, each $V_{j}$ is linear in the sense of Fishburn (1984) and, for all $q, q^{\prime} \in \mathcal{L}^{e}, V_{0}(q) \geq V_{0}\left(q^{\prime}\right)$ whenever $V_{i}(q) \geq V_{i}\left(q^{\prime}\right)$ for all $i \in N$ (by Pareto for equal risk and the definition of the $V_{i}$ functions). Hence, by Theorem 2 in Fishburn (1984), there exist non-negative real numbers $\left(\alpha_{i}\right)_{i \in N} \in \mathbb{R}^{n}$ and a real number $\beta$ such that, for all $q \in \mathcal{L}^{e}$ :

$$
V_{0}(q)=\sum_{i \in N} \alpha_{i} V_{i}(q)+\beta .
$$

Hence, there exist $\left(\alpha_{i}\right)_{i \in N} \in \mathbb{R}^{n}$ and $\beta \in \mathbb{R}$ such that for any $f \in \mathcal{F}^{e}$ :

$$
E(W \circ v \circ f)=V_{0}\left(q_{f}\right)=\sum_{i \in N} \alpha_{i} V_{i}\left(q_{f}\right)+\beta=\sum_{i \in N} \alpha_{i} E\left(u_{i} \circ f\right)+\beta .
$$

To sum up, there exist non-negative real numbers $\left(\alpha_{i}\right)_{i \in N}$ such that, for any $f, g \in$ $\mathcal{F}$,

$$
\begin{aligned}
f R_{\mathcal{F}} g & \Longleftrightarrow f^{e} R_{\mathcal{F}} g^{e} \\
& \Longleftrightarrow E\left(W \circ v \circ f^{e}\right) \geq E\left(W \circ v \circ g^{e}\right) \\
& \Longleftrightarrow \sum_{i \in N} \alpha_{i} E\left(u_{i} \circ f^{e}\right) \geq \sum_{i \in N} \alpha_{i} E\left(u_{i} \circ g^{e}\right) .
\end{aligned}
$$

\section{Proof of Theorem 2}

Suppose that Assumptions 3 and 5 hold, that Assumptions 1, 2 and 4 hold for each profile $\Phi$ (with $R_{W}^{\Phi}$ the relation in Assumption 2 for profile $\Phi$ ), and that each social 
ordering $R_{\mathcal{F}}^{\Phi}$ satisfies Agreement, Pareto for equal or no risk and Expected utility, as well as Invariance to risk attitudes for constant acts and Equity.

Expected utility implies that Dominance is satisfied and that $R_{W}^{\Phi}$ is continuous. For every $\Phi \in \mathcal{C}$ and $f \in \mathcal{F}$, denote $c e^{\Phi}(f)$ the certainty equivalent allocation of $f$ (which depends on risk attitudes) for the profile $\Phi$. By Proposition 2 and its Corollary 2, for all $\Phi \in \mathcal{C}$, for all $f, g \in \mathcal{F}$ :

$$
f R_{\mathcal{F}}^{\Phi} g \Longleftrightarrow c e^{\Phi}\left(f^{e}\right) R_{X}^{\Phi} c e^{\Phi}\left(g^{e}\right)
$$

Let $\Phi_{0}$ be a reference profile in $\mathcal{C}$ and denote $R_{X}:=R_{X}^{\Phi_{0}}$. By Invariance to risk attitudes for constant acts, we know that for all $\Phi \in \mathcal{C}$ and $f, g \in \mathcal{F}$ :

$$
c e^{\Phi}\left(f^{e}\right) R_{X}^{\Phi} c e^{\Phi}\left(g^{e}\right) \Longleftrightarrow c e^{\Phi}\left(f^{e}\right) R_{X} c e^{\Phi}\left(g^{e}\right)
$$

Hence, for any $\Phi \in \mathcal{C}$ and for all $f, g \in \mathcal{F}$ :

$$
f R_{\mathcal{F}}^{\Phi} g \Longleftrightarrow c e^{\Phi}\left(f^{e}\right) R_{X} c e^{\Phi}\left(g^{e}\right)
$$

For every $\Phi \in \mathcal{C}$, we also know by Theorem 1 that there exists a vector $\left(\alpha_{1}^{\Phi}, \cdots, \alpha_{n}^{\Phi}\right) \in$ $\mathbb{R}_{+}^{n}$ such that, for all $f, g \in \mathcal{F}$,

$$
f R_{\mathcal{F}}^{\Phi} g \Longleftrightarrow \sum_{i \in N} \alpha_{i}^{\Phi} E\left(u_{i} \circ f^{e}\right) \geq \sum_{i \in N} \alpha_{i}^{\Phi} E\left(u_{i} \circ g^{e}\right) .
$$

Define the set of allocations which are certainty-equivalent allocations of EDE acts:

$$
X^{\Phi}=\left\{x \in X \mid \exists f \in \mathcal{F}, x=c e^{\Phi}\left(f^{e}\right)\right\}
$$

Equations (A.1) and (A.2) imply that, for all $\Phi \in \mathcal{C}$ for all $x, y \in X^{\Phi}$ :

$$
x R_{X} y \Longleftrightarrow x R_{X}^{\Phi} y \Longleftrightarrow \sum_{i \in N} \alpha_{i}^{\Phi} u_{i}(x) \geq \sum_{i \in N} \alpha_{i}^{\Phi} u_{i}(y)
$$

Lemma A.1 For all $x, y \in X$, if there exist $\underline{v}, \bar{v} \in \mathbb{R}$ and $i \in N$ such that the following two conditions hold true:

1. $v_{j}(x)=\underline{v}$ and $v_{j}(y)=\bar{v}$ for all $j \in N \backslash i$;

2. $\inf V<v_{i}(y)<v_{i}(x) \leq \underline{v} \leq \bar{v}<\sup V$; 
then there exists $\Phi \in \mathcal{C}$ such that $x, y \in X^{\Phi}$ and

$$
\sum_{i \in N} \alpha_{i}^{\Phi} u_{i}(x)>\sum_{i \in N} \alpha_{i}^{\Phi} u_{i}(y)
$$

Proof. Let $x, y \in X$ satisfying the conditions in the Lemma and denote $\bar{u}=v_{i}(x)$ and $\underline{u}=v_{i}(y)$. Consider the following profile $\Phi \in \mathcal{C}$ :

1. For all $j \in N \backslash\{i\}, \phi_{j}(v)=v$ for all $v \in V$.

2. $\phi_{i}(v)= \begin{cases}\theta \kappa(v-\underline{u}) & \text { for all } v \leq \underline{u}, \\ \theta(v-\underline{u}) & \text { for all } \underline{u}<v \leq \bar{u}, \\ \theta(\bar{u}-\underline{u})+v-\bar{u} & \text { for all } v>\bar{u},\end{cases}$

where the values of $\kappa$ and $\theta$ will be set later.

\section{Step 1: Proof that $y \in X^{\Phi}$.}

By Assumption 5, for any $\hat{\pi} \in[0,1]$, there exists an event $\hat{A} \in \mathcal{A}$ such $p(\hat{A})=\hat{\pi}$. Because inf $V<\underline{u}, \bar{v}<\sup V$ and $V$ is an interval, there exists a small $\varepsilon>0$ such that $(\underline{u}-\varepsilon) \in V$ and $(\bar{v}+\varepsilon) \in V$. And it is always possible to find $\pi$ such that:

$$
\pi(\underline{u}-\varepsilon)+(1-\pi)(\bar{v}+\varepsilon)=\bar{v} .
$$

Indeed, $\pi=\varepsilon /(\bar{v}-\underline{u}+2 \varepsilon)$ satisfies this equation.

Let $A \in \mathcal{A}$ be a set such that $p(A)=\pi$. Then consider the following egalitarian act $f \in \mathcal{F}^{e}$ (which exists by Assumption 3). For all $\omega \in A, v_{k}(f(\omega))=\underline{u}-\varepsilon$ for all $k \in N$; and for all $\omega \in \Omega \backslash A, v_{k}(f(\omega))=\bar{v}+\varepsilon$ for all $k \in N$. To prove that $y \in X^{\Phi}$, we need to show that $v_{j}\left(c e^{\Phi}(f)\right)=\bar{v}$ for all $j \neq i$, and $v_{i}\left(c e^{\Phi}(f)\right)=\underline{u}$.

For any $j \neq i$, we have:

$$
E\left[\phi_{j}\left(v_{j}(f)\right)\right]=\pi(\underline{u}-\varepsilon)+(1-\pi)(\bar{v}+\varepsilon)=\phi_{j}(\bar{v}),
$$

because $\phi_{j}(v)=v$ for all $v$, and by definition of $\pi$. Hence, $v_{j}\left(c e^{\Phi}(f)\right)=\bar{v}^{24}$

For $i$, we have, by definition of $\phi_{i}$ :

$$
E\left[\phi_{i}\left(v_{i}(f)\right)\right]=-\pi \theta \kappa \varepsilon+(1-\pi)(\theta(\bar{u}-\underline{u})+\bar{v}-\bar{u}+\varepsilon) .
$$

Letting

$$
\kappa=\frac{(1-\pi)(\theta(\bar{u}-\underline{u})+\bar{v}-\bar{u}+\varepsilon)}{\pi \theta \varepsilon},
$$

${ }^{24}$ Indeed, if $E\left[u_{i} \circ f\right]=\phi_{i}(v)$, then by definition of the certainty equivalent, $v_{i}\left(c e^{\Phi}(f)\right)=v$. 
we obtain $E\left[\phi_{i}\left(v_{i}(f)\right)\right]=0=\phi_{i}(\underline{u})$, so that $v_{i}\left(c e^{\Phi}(f)\right)=\underline{u}$.

Step 2: Proof that $x \in X^{\Phi}$.

First note that, for every $0 \leq \widetilde{\varepsilon} \leq \varepsilon$, there exists $\pi(\widetilde{\varepsilon})$ such that:

$$
\pi(\widetilde{\varepsilon})(\underline{u}-\widetilde{\varepsilon})+(1-\pi(\widetilde{\varepsilon}))(\underline{v}+\widetilde{\varepsilon})=\underline{v} .
$$

Indeed, $\pi(\widetilde{\varepsilon})=\widetilde{\varepsilon} /(\underline{v}-\underline{u}+2 \widetilde{\varepsilon})$ satisfies this equation.

Let us now consider the function $h(\widetilde{\varepsilon})=\pi(\widetilde{\varepsilon}) \phi_{i}(\underline{u}-\widetilde{\varepsilon})+(1-\pi(\widetilde{\varepsilon})) \phi_{i}(\underline{v}+\widetilde{\varepsilon})$. This function is continuous. One has $\pi(0)=0$, so that $h(0)=\phi_{i}(\underline{v}) \leq \phi_{i}(\bar{v})$. On the other hand,

$$
\pi(\varepsilon)=\frac{\varepsilon}{\underline{v}-\underline{u}+2 \varepsilon} \geq \frac{\varepsilon}{\bar{v}-\underline{u}+2 \varepsilon}=\pi,
$$

because $\bar{v} \geq \underline{v}$. Given that $\underline{u}-\varepsilon<\bar{v}+\varepsilon$ and $\bar{v} \geq \underline{v}, h(\varepsilon) \leq \pi \phi_{i}(\underline{u}-\varepsilon)+(1-\pi) \phi_{i}(\bar{v}+\varepsilon)=$ $\phi_{i}(\underline{u})$, where the last equality comes from Step 1 .

Hence $h(\varepsilon) \leq \phi_{i}(\bar{u}) \leq h(0)$. By the intermediate value theorem, and because $\phi_{i}$ is continuous and increasing, there exists $0 \leq \bar{\varepsilon} \leq \varepsilon$ such that $h(\bar{\varepsilon})=\phi_{i}(\bar{u})$.

Let $A^{\prime}$ be a set such that $p\left(A^{\prime}\right)=\pi(\bar{\varepsilon})$. Then consider the following egalitarian act $g \in \mathcal{F}^{e}$. For all $\omega \in A^{\prime}, v_{k}(g(\omega))=\underline{u}-\bar{\varepsilon}$ for all $k \in N$; and for all $\omega \in \Omega \backslash A^{\prime}$, $v_{k}(g(\omega))=\underline{v}+\bar{\varepsilon}$ for all $k \in N$. To prove that $x \in X^{\Phi}$, we need to show that $v_{j}\left(c e^{\Phi}(g)\right)=\underline{v}$ for all $j \neq i$, and $v_{i}\left(c e^{\Phi}(g)\right)=\bar{u}$.

For all $j \neq i$, we have:

$$
E\left[\phi_{j}\left(v_{j}(g)\right)\right]=\pi(\bar{\varepsilon})(\underline{u}-\bar{\varepsilon})+(1-\pi(\bar{\varepsilon}))(\underline{v}+\bar{\varepsilon})=\phi_{j}(\underline{v}),
$$

because $\phi_{j}(v)=v$ for all $v$, and by definition of $\pi(\bar{\varepsilon})$. Hence, $v_{j}\left(c e^{\Phi}(g)\right)=\underline{v}$.

For $i$, we have $E\left[\phi_{i}\left(v_{i}(g)\right)\right]=h(\bar{\varepsilon})=\phi_{i}(\bar{u})$, therefore $v_{i}\left(c e^{\Phi}(g)\right)=\bar{u}$.

Step 3: Proof that $(n-1) \alpha_{i}^{\Phi} \geq \sum_{j \neq i} \alpha_{j}^{\Phi}$.

Let $\widetilde{x}, \widetilde{y} \in X$ be such that for some $0<\varepsilon<(\bar{v}-\underline{v}) / n,{ }^{25}$

1. $v_{j}(\widetilde{y})=\bar{v}$ and $v_{j}(\widetilde{x})=\bar{v}-\varepsilon$ for all $j \in N \backslash i$;

2. $v_{i}(\widetilde{y})=\underline{v}$ and $v_{i}(\widetilde{x})=\underline{v}+(n-1) \varepsilon$.

${ }^{25}$ Such $\widetilde{x}, \widetilde{y} \in X$ exist by Assumption 3 . 
By reasonings similar to those in Step 1 (for $\widetilde{y}$ ) and Step 2 (for $\widetilde{x}$ ), it is possible to show that $\widetilde{y}, \widetilde{x} \in X^{\Phi}$. By $(n-1)$ successive applications of the Equity principle, we also know that $\widetilde{x} R_{X}^{\Phi} \widetilde{y}$. By Equation (A.3), this implies that:

$$
\sum_{i \in N} \alpha_{i}^{\Phi} \phi_{i}\left(v_{i}(\widetilde{x})\right) \geq \sum_{i \in N} \alpha_{i}^{\Phi} \phi_{i}\left(v_{i}(\widetilde{y})\right)
$$

By definition of $\Phi, \widetilde{x}$ and $\widetilde{y}$, this yield $\alpha_{i}^{\Phi}(n-1) \varepsilon \geq \sum_{j \neq i} \alpha_{j}^{\Phi} \varepsilon$. Now cancel $\varepsilon$ from both sides to prove that $\alpha_{i}^{\Phi}(n-1) \geq \sum_{j \neq i} \alpha_{j}^{\Phi}$.

\section{Step 4: Conclusion.}

We have:

$$
\begin{aligned}
\sum_{j \in N} \alpha_{j}^{\Phi} \phi_{j}\left(v_{i}(x)\right) & =\alpha_{i}^{\Phi} \theta(\bar{u}-\underline{u})+\left(\sum_{j \neq i} \alpha_{j}^{\Phi}\right) \underline{v} \\
& =\left(\sum_{j \neq i} \alpha_{j}^{\Phi}\right) \bar{v}+\left(\alpha_{i}^{\Phi} \theta(\bar{u}-\underline{u})-\left(\sum_{j \neq i} \alpha_{j}^{\Phi}\right)(\bar{v}-\underline{v})\right) \\
& \geq \sum_{j \in N} \alpha_{j}^{\Phi} \phi_{j}\left(v_{j}(y)\right)+\left(\sum_{j \neq i} \alpha_{j}^{\Phi}\right)\left(\frac{\theta(\bar{u}-\underline{u})}{n-1}-(\bar{v}-\underline{v})\right) .
\end{aligned}
$$

Let us set $\theta>(n-1) \frac{\bar{v}-\underline{v}}{\bar{u}-\underline{u}}$. Hence, we obtain:

$$
\sum_{i \in N} \alpha_{i}^{\Phi} \phi_{i}\left(v_{i}(x)\right)>\sum_{i \in N} \alpha_{i}^{\Phi} \phi_{i}\left(v_{i}(y)\right)
$$

and therefore

$$
\sum_{i \in N} \alpha_{i}^{\Phi} u_{i}(x)>\sum_{i \in N} \alpha_{i}^{\Phi} u_{i}(y)
$$

\section{Proof of implication 1.}

Consider any $x, y \in X$ such that $\min _{i \in N} v_{i}(x)>\min _{i \in N} v_{i}(y)$. Let $i^{*}$ be such that $v_{i^{*}}(x)=\min _{i \in N} v_{i}(x), \underline{u}=\min _{i \in N} v_{i}(y), \bar{u}=\min _{i \in N} v_{i}(x), \underline{v}=\min _{i \in N \backslash i^{*}} v_{i}(x), \bar{v}=$ $\max _{i \in N} v_{i}(y)$. Hence, $\underline{u}$ is the well-being of the worst-off individual in $y, \bar{u}$ is the well-being of the worst-off individual in $x, \underline{v}$ is the well-being of the second worst-off individual in $x, \bar{v}$ is the well-being of the best-off individual in $y$. Define $\bar{x}, \bar{y} \in X$ in the following way (it is possible to do so by Assumption 3): there exists $i \in N$ such that 
1. $v_{j}(\bar{x})=\underline{v}$ and $v_{j}(\bar{y})=\bar{v}$ for all $j \in N \backslash i$;

2. $v_{i}(\bar{y})=\underline{u}, v_{i}(\bar{x})=\bar{u}$.

By Assumption 2, the social welfare ordering $R_{W}$ associated to $R_{X}$ is weakly monotonic, and we necessarily have $x R_{X} \bar{x}$ and $\bar{y} R_{X} y$. By the same token, if $\underline{v}>\bar{v}$, we have that $\bar{x} P_{X} \bar{y}$, which by transitivity implies $x P_{X} y$.

Let us thus consider the case $\underline{v} \leq \bar{v}$. If $\inf V<\underline{u}$ and $\bar{v}<\sup V$, allocations $\bar{x}$ and $\bar{y}$ satisfy the conditions of Lemma A.1. ${ }^{26}$ Thus there exists $\bar{\Phi} \in \mathcal{C}$ such that $\bar{x}, \bar{y} \in X^{\bar{\Phi}}$ and

$$
\sum_{i \in N} \alpha_{i}^{\bar{\Phi}} u_{i}(\bar{x})>\sum_{i \in N} \alpha_{i}^{\bar{\Phi}} u_{i}(\bar{y})
$$

But, by Equation (A.3), since $\bar{x}, \bar{y} \in X^{\bar{\Phi}}$, this implies that $\bar{x} P_{X} \bar{y}$. By transitivity, this implies $x P_{X} y$ and hence, by Invariance to risk attitudes for constant acts, $x P_{X}^{\Phi} y$ for all $\Phi \in \mathcal{C}$.

The only remaining case to consider is $\min _{i \in N} v_{i}\left(x_{i}\right)=\min _{i \in N} v_{i}\left(y_{i}\right)$. This case can be handled by continuity of $R_{W}$ to show that $x I_{X} y$ and hence $x I_{X}^{\Phi} y$ for all $\Phi \in \mathcal{C}$.

\section{Proof of implication 2.}

By Equation (A.1), for any $\Phi \in \mathcal{C}$ and for all $f, g \in \mathcal{F}$ :

$$
f R_{\mathcal{F}}^{\Phi} g \Longleftrightarrow c e^{\Phi}\left(f^{e}\right) R_{X} c e^{\Phi}\left(g^{e}\right) .
$$

By implication 1 of the theorem, this implies that for any $\Phi \in \mathcal{C}$ and for all $f, g \in \mathcal{F}$ :

$$
f R_{\mathcal{F}}^{\Phi} g \Longleftrightarrow \min _{i \in N} v_{i}\left(c e^{\Phi}\left(f^{e}\right)\right) \geq \min _{i \in N} v_{i}\left(c e^{\Phi}\left(g^{e}\right)\right) .
$$

By definition of the certainty-equivalent, for all $i \in N$ and for all $f \in \mathcal{F}, u_{i}\left(c e^{\Phi}\left(f^{e}\right)\right)=$ $E\left(u_{i} \circ f^{e}\right)$. Given that $u_{i}=\phi_{i} \circ v_{i}$ and because $\phi_{i}$ is increasing, we know that $v_{i}\left(c e^{\Phi}\left(f^{e}\right)\right)=$ $\phi_{i}^{-1}\left(E\left(u_{i} \circ f^{e}\right)\right)$.

By implication 1 of the theorem, we also know that for all $x \in X, v_{i}\left(x^{e}\right)=$ $\min _{j \in N} v_{j}(x)$ for all $i \in N$. Hence, for all $f \in \mathcal{F}$ and all $\omega \in \Omega, v_{i}\left(f^{e}(\omega)\right)=$ $\min _{j \in N} v_{j}(f(\omega))$ for all $i \in N$, so that

$$
u_{i}\left(f^{e}(\omega)\right)=\phi_{i} \circ v_{i}\left(f^{e}(\omega)\right)=\phi_{i}\left(\min _{j \in N} v_{j}(f(\omega))\right),
$$

\footnotetext{
${ }^{26}$ Other cases can be handled by continuity of $R_{X}$.
} 
for all $i \in N$.

Therefore, for all $f \in \mathcal{F}$ and $i \in N$,

$$
v_{i}\left(c e^{\Phi}\left(f^{e}\right)\right)=\phi_{i}^{-1}\left[\int_{\Omega} \phi_{i}\left(\min _{j} v_{j}(f(\omega))\right) d p(\omega)\right] .
$$

Hence, for all $f, g \in \mathcal{F}$, for all $\Phi \in \mathcal{C}$ :

$$
\begin{aligned}
f R_{\mathcal{F}}^{\Phi} g \Longleftrightarrow \min _{i} \phi_{i}^{-1}\left[\int_{\Omega} \phi_{i}\left(\min _{j} v_{j}(f(\omega))\right) d p(\omega)\right] \\
\quad \geq \min _{i} \phi_{i}^{-1}\left[\int_{\Omega} \phi_{i}\left(\min _{j} v_{j}(g(\omega))\right) d p(\omega)\right] .
\end{aligned}
$$

\section{Proof of Proposition 3}

Suppose that Assumptions 2, 3 and 6 hold.

By Assumption 2, the $v_{i}$ functions are ordinally equivalent to the $U_{i}$ functionals on riskfree allocations. Therefore, there exist increasing functions $\hat{\phi}_{i}$ such that $U_{i}(x)=$ $\hat{\phi}_{i} \circ v_{i}(x)$ for all $x \in X$. By definition of the certainty-equivalent (Assumption 6), we have $U_{i}(c e(f))=U_{i}(f)$ for all $f \in \mathcal{F}$, so that $v_{i}(c e(f))=\hat{\phi}_{i}^{-1} \circ U_{i}(f)$ and, for all $f, g \in \mathcal{F}$, and for all $i \in N$ :

$$
f R_{i} g \Longleftrightarrow v_{i}(c e(f)) \geq v_{i}(c e(g))
$$

Assume that the social ordering $R_{\mathcal{F}}$ satisfies Agreement, Dominance and Pareto for equal risk. We can proceed like in Step 1 of the proof of Proposition 1 to show that there exists a weakly monotonic ordering $\succsim^{*}$ of $Z=\left\{z \in \mathbb{R}^{n} \mid \exists f \in \mathcal{F}^{e}, z=\left(v_{i}(c e(f))\right)_{i \in N}\right\}$ such that for all $f, g \in \mathcal{F}^{e}: 27$

$$
\left(v_{i}(c e(f))\right)_{i \in N} \succsim^{*}\left(v_{i}(c e(g))\right)_{i \in N} \Longleftrightarrow f R_{\mathcal{F}} g .
$$

If $R_{\mathcal{F}}$ satisfies Pareto for equal risk, we can then proceed as in Steps 2 and 3 of the proof of Proposition 1 to prove statement 1 of Proposition 3.

If $R_{\mathcal{F}}$ satisfies Pareto for equal or no risk, we can then proceed exactly as in the proof of Proposition 2 to prove statement 2 of Proposition 3.

\footnotetext{
${ }^{27}$ Like in Proposition $1, \succsim^{*}$ is defined on $Z$ by $z \succsim^{*} z^{\prime}$ if there exists $f, g \in \mathcal{F}^{e}$ such that $f R_{\mathcal{F}} g$, $z=\left(v_{i}(c e(f))\right)_{i \in N}$ and $z^{\prime}=\left(v_{i}(c e(g))\right)_{i \in N}$.
} 


\section{Proof of Proposition 4}

Suppose that Assumptions 3 and 5 hold, that Assumptions 2, 6 and 7 hold for each profile $\Phi$, and that each social ordering $R_{\mathcal{F}}^{\Phi}$ satisfies Agreement, Pareto for equal or no risk, Invariance to uncertainty attitudes for constant acts, Equity and Expected utility on $\overline{\mathcal{F}}$.

By Proposition 3 and continuity of $R_{W}^{\Phi}$, we obtain (as in Corollary 2) that for all $f, g \in \mathcal{F}, f R_{\mathcal{F}}^{\Phi} g$ if and only if

$$
c e\left(f^{e}\right) R_{X}^{\Phi} c e\left(g^{e}\right) .
$$

Restricting $R_{\mathcal{F}}^{\Phi}$ to $\overline{\mathcal{F}}$, we can apply Theorem 2 and find that for all $x, y \in X$, for all $\Phi \in \mathcal{D}:$

$$
x R_{X}^{\Phi} y \Longleftrightarrow \min _{i \in N} v_{i}(x) \geq \min _{i \in N} v_{i}(y)
$$

which is part 1 of the Proposition.

By Equation (A.5), it must then be the case that for all $f, g \in \mathcal{F}, f R_{\mathcal{F}}^{\Phi} g$ if and only if

$$
\min _{i \in N} v_{i}\left(c e\left(f^{e}\right)\right) \geq \min _{i \in N} v_{i}\left(c e\left(f^{e}\right)\right)
$$

But, by definition of maxmin expected utilities, $u_{i}\left(c e\left(f^{e}\right)\right)=\min _{q \in \mathcal{Q}_{i}} E_{q}\left(u_{i} \circ f^{e}\right)$. This implies that

$$
v_{i}\left(c e\left(f^{e}\right)\right)=\phi_{i}^{-1}\left(\min _{q \in \mathcal{Q}_{i}} E_{q}\left(u_{i} \circ f^{e}\right)\right)=\phi_{i}^{-1}\left(\min _{q \in \mathcal{Q}_{i}} E_{q}\left[\phi_{i}\left(\min _{j} v_{j} \circ f\right)\right]\right),
$$

because, by equivalence (A.6), $u_{i}\left(f^{e}(\omega)\right)=\phi_{i}\left(\min _{j} v_{j}(f(\omega))\right)$ for all $i \in N$ and $\omega \in \Omega$.

Let $\bar{\psi}$ be the most concave function among $\left(\phi_{1}, \cdots, \phi_{n}\right)$. For all $i \in N$, we have $\phi_{i}^{-1}\left(\min _{q \in \mathcal{Q}_{i}} E_{q}\left[\phi_{i}\left(\min _{j} v_{j} \circ f\right)\right]\right) \geq \bar{\psi}^{-1}\left(\min _{q \in \mathcal{Q}_{i}} E_{q}\left[\bar{\psi}\left(\min _{j} v_{j} \circ f\right)\right]\right)$ (because $\bar{\psi}$ is at least as concave as $\phi_{i}$ ). Also, by definition of $\mathcal{D}$, there exists $j \in N$ such that $v_{j}\left(c e\left(f^{e}\right)\right)=\bar{\psi}^{-1}\left(\min _{q \in \mathcal{Q}_{i}} E_{q}\left[\bar{\psi}\left(\min _{j} v_{j} \circ f\right)\right]\right)$. Hence,

$\min _{i \in N} v_{i}\left(c e\left(f^{e}\right)\right)=\min _{i \in N} \bar{\psi}^{-1}\left(\min _{q \in \mathcal{Q}_{i}} E_{q}\left[\bar{\psi}\left(\min _{j} v_{j} \circ f\right)\right]\right)=\bar{\psi}^{-1}\left(\min _{i \in N} \min _{q \in \mathcal{Q}_{i}} E_{q}\left[\bar{\psi}\left(\min _{j} v_{j} \circ f\right)\right]\right)$,

where the last equality comes from the fact that $\bar{\psi}^{-1}$ is increasing.

For all $q \in \cup_{i \in N} \mathcal{Q}_{i}, E_{q}\left[\bar{\psi}\left(\min _{j} v_{j} \circ f\right)\right] \geq \min _{i \in N} \min _{q \in \mathcal{Q}_{i}} E_{q}\left[\bar{\psi}\left(\min _{j} v_{j} \circ f\right)\right]$, so that $\min _{i \in N} \min _{q \in \mathcal{Q}_{i}} E_{q}\left[\bar{\psi}\left(\min _{j} v_{j} \circ f\right)\right]=\min _{q \in \cup_{i \in N} \mathcal{Q}_{i}} E_{q}\left[\bar{\psi}\left(\min _{j} v_{j} \circ f\right)\right]$. 
Hence, for all $f, g \in \mathcal{F}$,

$$
\begin{aligned}
f R_{\mathcal{F}}^{\Phi} g & \Longleftrightarrow \min _{i \in N} v_{i}\left(c e\left(f^{e}\right)\right) \geq \min _{i \in N} v_{i}\left(c e\left(g^{e}\right)\right) \\
& \Longleftrightarrow \bar{\psi}^{-1}\left(\min _{q \in \cup_{i \in N} \mathcal{Q}_{i}} E_{q}\left[\bar{\psi}\left(\min _{j} v_{j} \circ f\right)\right]\right) \geq \bar{\psi}^{-1}\left(\min _{q \in \cup_{i \in N} \mathcal{Q}_{i}} E_{q}\left[\bar{\psi}\left(\min _{j} v_{j} \circ g\right)\right]\right) \\
& \Longleftrightarrow \min _{q \in \cup_{i \in N} \mathcal{Q}_{i}} E_{q}\left[\bar{\psi}\left(\min _{j} v_{j} \circ f\right)\right] \geq \min _{q \in \cup_{i \in N} \mathcal{Q}_{i}} E_{q}\left[\bar{\psi}\left(\min _{j} v_{j} \circ g\right)\right] .
\end{aligned}
$$

\section{Appendix B: Optimal unemployment allowance policy under macroe- conomic risk}

We first present the original problem studied by Gollier (1991):

$$
\begin{array}{ll}
\max & \int_{0}^{+\infty} \lambda(m)\left[\int_{\Omega} \phi(\max \{W(\omega)-m, B(\omega)\}) d p(\omega)\right] d G(m) \\
\text { s.t. } & \int_{\Omega}[F(L(\omega), \omega)-W(\omega) L(\omega)-(1-L(\omega)) B(\omega)] d p(\omega) \geq \bar{v} \\
& L(\omega)=G(W(\omega)-B(\omega)) \forall \omega \\
& F_{1}(L(\omega), \omega)=W(\omega)-B(\omega) \forall \omega
\end{array}
$$

The Lagrangian of this problem can be written as

$$
\begin{aligned}
& \mathcal{L}= \int_{\Omega}\left[\int_{0}^{W(\omega)-B(\omega)} \lambda(m) \phi(W(\omega)-m) d G(m)\right. \\
&\left.+\int_{W(\omega)-B(\omega)}^{+\infty} \lambda(m) \phi(B(\omega)) d G(m)\right] d p(\omega) \\
&+\gamma \int_{\Omega}[F(G(W(\omega)-B(\omega)), \omega)\quad-W(\omega) G(W(\omega)-B(\omega))-[1-G(W(\omega)-B(\omega))] B(\omega)-\bar{v}] d p(\omega) \\
&+\int_{\Omega} \eta(\omega)\left[F_{1}(G(W(\omega)-B(\omega)), \omega)-W(\omega)+B(\omega)\right] d p(\omega)
\end{aligned}
$$

And the first order conditions for an interior solution are: ${ }^{28}$

$$
\begin{aligned}
\frac{\partial \mathcal{L}}{\partial W(\omega)} & =\int_{0}^{W(\omega)-B(\omega)} \lambda(m) \phi^{\prime}(W(\omega)-m) d G(m)-\zeta(\omega)=0 \\
\frac{\partial \mathcal{L}}{\partial B(\omega)} & =\int_{W(\omega)-B(\omega)}^{+\infty} \lambda(m) \phi^{\prime}(B(\omega)) d G(m)-\gamma+\zeta(\omega)=0,
\end{aligned}
$$

\footnotetext{
${ }^{28}$ There are important simplifications in the FOC. First, the derivatives with respect to the integral bounds in $\int_{0}^{W(\omega)-B(\omega)}$ and $\int_{W(\omega)-B(\omega)}^{+\infty}$ cancel out because for $m=W(\omega)-B(\omega)$ one has $\phi(W(\omega)-m)=\phi(B(\omega))$. Second, in the first constraint, the factor of $G^{\prime}(W(\omega)-B(\omega))$ is $F_{1}()-.W(\omega)+B(\omega) \equiv 0$.
} 
where $^{29}$

$$
\begin{aligned}
\zeta(\omega)= & \gamma G(W(\omega)-B(\omega)) \\
& -\eta(\omega) F_{11}(G(W(\omega)-B(\omega)), \omega) G^{\prime}(W(\omega)-B(\omega))+\eta(\omega) .
\end{aligned}
$$

Equations (B.4) and (B.5) imply

$$
\int_{0}^{W(\omega)-B(\omega)} \lambda(m) \phi^{\prime}(W(\omega)-m) d G(m)+\int_{W(\omega)-B(\omega)}^{+\infty} \lambda(m) \phi^{\prime}(B(\omega)) d G(m)=\gamma .
$$

Denote

$$
E D E(\omega)=\varphi^{-1}\left(\int_{0}^{+\infty} \lambda(m) \varphi(\max \{W(\omega)-m, B(\omega)\}) d G(m)\right) .
$$

We now consider the alternative problem corresponding to the social welfare ordering suggested in Theorem 1.

$$
\begin{array}{ll}
\max & \int_{\Omega} \phi(E D E(\omega)) d p(\omega) \\
\text { s.t. } & (B .1),(B .2),(B .3) .
\end{array}
$$

Proceeding as before, we can show that the first order conditions for an interior solution of the problem are:

$$
\begin{aligned}
\frac{\partial \mathcal{L}}{\partial W(\omega)} & =\frac{\phi^{\prime}(E D E(\omega))}{\varphi^{\prime}(E D E(\omega))}\left(\int_{0}^{W(\omega)-B(\omega)} \lambda(m) \phi^{\prime}(W(\omega)-m) d G(m)\right)-\zeta(\omega)=0 \\
\frac{\partial \mathcal{L}}{\partial B(\omega)} & =\frac{\phi^{\prime}(E D E(\omega))}{\varphi^{\prime}(E D E(\omega))}\left(\int_{W(\omega)-B(\omega)}^{+\infty} \lambda(m) \phi^{\prime}(B(\omega)) d G(m)\right)-\gamma+\zeta(\omega)=0,
\end{aligned}
$$

where $\zeta(\omega)$ is defined as before. This implies that

$$
\frac{\phi^{\prime}(E D E(\omega))}{\varphi^{\prime}(E D E(\omega))}\left(\int_{0}^{W(\omega)-B(\omega)} \lambda(m) \phi^{\prime}(W(\omega)-m) d G(m)+\int_{W(\omega)-B(\omega)}^{+\infty} \lambda(m) \phi^{\prime}(B(\omega)) d G(m)\right)=\gamma
$$

When $\varphi$ becomes infinitely concave (i.e. when an infinite priority is given to the worst-off), the equally-distributed equivalent tends to $E D E(\omega)=\min _{i \in N} \max \{W(\omega)-$ $\left.m_{i}, B(\omega)\right\}=B(\omega)$. The social maximization program then becomes:

$$
\begin{array}{ll}
\max & \int_{\Omega} \phi(B(\omega)) d p(\omega) \\
\text { s.t. } & (B .1),(B .2),(B .3),
\end{array}
$$

\footnotetext{
${ }^{29} F_{11}$ denotes the second derivative of $F$ with respect to $L$.
} 
so that the first order conditions are simply

$$
\begin{aligned}
\frac{\partial \mathcal{L}}{\partial W(\omega)} & =-\zeta(\omega)=0 \\
\frac{\partial \mathcal{L}}{\partial B(\omega)} & =\phi^{\prime}(B(\omega))-\gamma+\zeta(\omega)=0
\end{aligned}
$$

implying $\phi^{\prime}(B(\omega))=\gamma$.

\section{References}

Adler, M.D., 2012. Well-Being and Fair Distribution: Beyond Cost-Benefit Analysis. Oxford University Press, Oxford.

Al-Najjar, N.I., Weinstein, J., 2009. The ambiguity aversion literature: A critical assessment. Econ. Philos. 25, 249-284.

Asheim, G.B., Zuber, S., 2016. Evaluating intergenerational risks. J. Math. Econ. 65, 104-117.

Blackorby, C., Donaldson, D., Weymark, J.A., 1999. Harsanyi's social aggregation theorem for state-contingent alternatives. J. Math. Econ. 32, 365-387.

Broome, J., 1991. Weighing Goods - Equality, Uncertainty and Time. Blackwell, Oxford.

Cerreia-Vioglio, S., Ghirardato, P., Maccheroni, F., Marinacci, M., Siniscalchi, M., 2011. Rational preferences under ambiguity. Econ. Theory 48, 341-375.

Chambers, C.P., Echenique, E., 2012. When does aggregation reduce risk aversion? Games Econ. Behav. 76, 582-595.

Chambers, C.P., Hayashi, T., 2006. Preference aggregation under uncertainty: Savage vs. Pareto. Games Econ. Behav. 54, 430-440.

Chew, C.S. Sagi, J.S., 2012. An inequality measure for stochastic allocations. J. Econ. Theory $147,1517-1544$.

Cres, H., Gilboa, I., Vieille, N., 2011. Aggregation of multiple prior opinions. J. Econ. Theory $146,2563-2582$.

Danan, E., Gajdos, T., Hill, B., Tallon, J.-M., 2016. Robust social decisions. American Econ. Rev. 106, 2407-2425.

Debreu, G., 1959. Theory of Value - An Axiomatic Analysis of Economic Equilibrium. Yale University Press, New Haven.

Decancq, K., Fleurbaey, M., Schokkaert, E., 2015. Inequality, income and well-being. In: Atkinson, A.B., Bourguignon, F. (Eds.), Handbook of Income Distribution. North Holland, Amsterdam, pp. 67-140.

Decerf, B., Van der Linden, M., 2016. Fair social orderings with other-regarding preferences. Soc. Choice Welfare 46, 655-694.

Dhillon, A., Mertens, J.-F., 1999. Relative utilitarianism. Econometrica 67, 471-498.

Diamond, P.A., 1967. Cardinal welfare, individualistic ethics, and interpersonal comparison of utility: Comment. J. Polit. Economy 75, 765-766.

Drèze, J., Gollier, C., 1993. Risk sharing on the labour market and second-best wage rigidities. Europ. Econ. Rev. 37, 1457-1482.

Drummond, M.F., Sculpher, M.J., Torrance, G.W., O’Brien, B.L., Stoddart, G.L., 2005. Methods for the Economic Evaluation of Health Care Programmes. Oxford University Press, Oxford. 
Dufwenberg, M., Heidhues, P., Kirchsteiger, G., Riedel, F., Sobel, J., 2001. Other-regarding preferences in general equilibrium. Rev. Econ. Stud. 78, 613-639.

Epstein, L.G., Segal, U., 1992. Quadratic social welfare functions. J. Polit. Economy 100, 691712 .

Fishburn, P.C., 1984. On Harsanyi's utilitarian cardinal welfare theorem. Theory Dec. 17, 2128.

Fleurbaey, M., 2010. Assessing risky social situations. J. Polit. Economy 118, 649-680.

Fleurbaey, M., Gajdos, T., Zuber, S., 2015. Social rationality, separability, and equity under uncertainty, Math. Soc. Sci. 73, 13-22.

Fleurbaey, M., Maniquet, F., 2011. A Theory of Fairness and Social Welfare. Cambridge University Press, Cambridge.

Fleurbaey, M., Mongin, P., 2016. The utilitarian relevance of Harsanyi's theorem. American Econ. J.: Microeconomics 8, 289-306.

Fleurbaey, M., Zuber, S., 2016. Fair intergenerational decision-making. Mimeo.

Gajdos, T., Maurin, E., 2004. Unequal uncertainties and uncertain inequalities: An axiomatic approach. J. Econ. Theory 116, 93-118.

Gajdos, T., Tallon, J.-M., 2002. Fairness under uncertainty. Econ. Bulletin 18, 1-7.

Gilboa, I., 2015. Rationality and the Bayesian paradigm. J. Econ. Methodo. 22, 312-334.

Gilboa, I., Samet, D., 0 Schmeidler, D., 2004. Utilitarian aggregation of beliefs and tastes. J. Polit. Economy 112, 932-938.

Gilboa, I., Schmeidler, D., 1989. Maxmin expected utility with non-unique prior. J. Math. Econ. $18,141-153$.

Gollier, C., 1991. Unemployment insurance: Risk sharing versus efficiency. Geneva Risk Insur. Rev. 16, 59-74.

Grant, S., Kajii, A., Polak, B., Safra, Z., 2010. Generalized utilitarianism and Harsanyi's impartial observer theorem. Econometrica 78, 1939-1971.

Harsanyi, J.C., 1955. Cardinal welfare, individualistic ethics and interpersonal comparisons of utility. J. Polit. Economy 63, 309-321.

Heal, G., Millner, A., 2015. Should climate policy account for ambiguity? Mimeo.

Karni, E., 1998. Impartiality: Definition and representation. Econometrica 66, 1405-1415.

Mongin, P., 1995. Consistent Bayesian aggregation. J. Econ. Theory 66, 313-351.

Mongin, P., 1998. The paradox of the Bayesian expert and state-dependent utility theory. J. Math. Econ. 29, 331-361.

Mongin, P., 2016. Spurious unanimity and the Pareto principle. Econ. Philos. 32, 511-532.

Mongin, P., Pivato, M., 2016. Social preference under twofold uncertainty. Mimeo.

Millner, A., 2013. On welfare frameworks and catastrophic climate risks. J. Environ. Econ. Manage. 65, 310-325.

Nascimento, L., 2012. The ex ante aggregation of opinions under uncertainty. Theoretical Econ. 7, 535-570.

Pazner, E., 1979. Equity, nonfeasible alternatives and social choice: A reconsideration of the concept of social welfare. In: Laffond, J.-J. (Ed.), Aggregation and Revelation of Preferences. North-Holland, Amsterdam, pp. 161-173.

Pindyck, R.S., Wang, N., 2009. The economic and policy consequences of catastrophes. American Econ. J.: Econ. Pol. 5, 306-339. 
Qu, X. (2015). Separate aggregation of beliefs and values under ambiguity. Forthcoming in Econ. Theory, doi:10.1007/s00199-015-0944-1.

Rawls, J., 1971. A Theory of Justice, 1999 revised ed. Harvard University Press, Cambridge.

Savage, L.J., 1972. The Foundations of Statistics, 2nd revised ed. Dover Publications, New York.

Segal, E., 1998. Let's agree that all dictatorships are equally bad. J. Polit. Economy 108, 569589.

Sen, A.K., 1976. Welfare inequalities and Rawlsian axiomatics. Theory Dec. 7, 243-262.

Sen, A.K., 1985. Commodities and Capabilities. New-Holland, Amsterdam.

Treibich, R., 2015. Welfare egalitarianism with other-regarding preferences. Mimeo.

Weitzman, M.L., 2009. On modeling and interpreting the economics of catastrophic climate change. Rev. Econ. Stat. 91, 1-19.

Weymark, J.A., 1991. A reconsideration of the Harsanyi-Sen debate on utilitarianism. In: Elster, J., Roemer, J.E. (Eds.), Interpersonal Comparisons of Well-Being. Cambridge University Press, Cambridge, pp. 255-320. 